\title{
The nuclear activity of the actin-binding Moesin protein is necessary for gene expression in Drosophila
}

\author{
Csaba Bajusz ${ }^{1,2}$, Ildikó Kristó ${ }^{1}$, Csilla Abonyi ${ }^{1}$, Tomáš Venit ${ }^{3}$, Viktor Vedelek ${ }^{4}$, Tamás Lukácsovich ${ }^{5}$, \\ Attila Farkas ${ }^{1}$, Péter Borkúti ${ }^{1,6}$, Zoltán Kovács ${ }^{1,6}$, Izabella Bajusz ${ }^{1}$, Annamária Marton ${ }^{1}$, \\ Csaba Vizler ${ }^{1}$, Zoltán Lipinszki ${ }^{7}$, Rita Sinka ${ }^{4}$, Piergiorgio Percipalle ${ }^{3,8}$ and Péter Vilmos ${ }^{1}$ \\ 1 Eötvös Loránd Research Network (ELKH), Biological Research Centre, Szeged, Hungary \\ 2 Doctoral School of Biology, University of Szeged, Hungary \\ 3 Biology Program, Science Division, New York University Abu Dhabi, UAE \\ 4 Department of Genetics, University of Szeged, Hungary \\ 5 Brain Research Institute, University of Zürich, Switzerland \\ 6 Doctoral School of Multidisciplinary Medical Science, University of Szeged, Hungary \\ 7 Lendület Laboratory of Cell Cycle Regulation, ELKH, Biological Research Centre, Szeged, Hungary \\ 8 Department of Molecular Biosciences, The Wenner-Gren Institute, Stockholm University, Sweden
}

\author{
Keywords \\ Drosophila; Moesin; nucleus; Hsp; \\ transcription

\section{Correspondence \\ P. Vilmos, Biological Research Centre, Szeged 6726, Temesvári krt. 62, Hungary Tel: +36-62-599689 \\ E-mail: vilmosp@brc.hu}

(Received 19 September 2020, revised 22 January 2021, accepted 18 February 2021)

doi:10.1111/febs.15779
Ezrin-Radixin-Moesin (ERM) proteins play an essential role in the cytoplasm by cross-linking actin filaments with plasma membrane proteins. Research has identified the nuclear localization of ERMs, as well as the involvement of a single Drosophila ERM protein, Moesin, in nuclear mRNA exports. However, the question of how important the nuclear activity of ERM proteins are for the life of an organism has so far not been explored. Here, we present the first attempt to reveal the in vivo relevance of nuclear localization of Moesin in Drosophila. With the help of a nuclear export signal, we decreased the amount of Moesin in the nuclei of the animals. Furthermore, we observed various developmental defects, demonstrating the importance of ERM function in the nucleus for the first time. Transcriptome analysis of the mutant flies revealed that the lack of nuclear Moesin function leads to expression changes in nearly 700 genes, among them heat-shock genes. This result together with additional findings revealed that in Drosophila the expression of protein chaperones requires the nuclear functions of Moesin.

Database

GEO accession number: GSE155778.

\section{Introduction}

The presence of cytoskeletal proteins in the nucleus is undisputed today, and it is now clear that they are involved in fundamental nuclear functions [1-4]. However, the biological relevance of the activity of cytoskeletal proteins in the nucleus has not yet been determined and is still debated. This is primarily due to the fact that cytoskeletal proteins have essential activities in the cytoplasm, and therefore, the elimination or inhibition of these proteins affects their activity in the cytoplasm as well. Furthermore, with the exception of actin, the mechanism of their nuclear transport has not been resolved. Therefore, the investigation of

\footnotetext{
Abbreviations

CRM1, chromosomal region maintenance 1; ERM, Ezrin-Radixin-Moesin; FCS, fetal calf serum; GO, gene ontology; hsp, heat-shock protein; Moe, Moesin; NES, nuclear export signal; PCA, principal component analysis; RT, room temperature; WGA, wheat germ agglutinin; WT, wild-type.
} 
their nuclear activity separately from their cytoplasmic functions has been an enormous challenge.

The evolutionarily highly conserved family of the actin-binding, cytoskeletal ERM (ezrin, radixin, and moesin) proteins consists of three closely related paralogs: ezrin, radixin, and moesin [5,6]. They are all present in vertebrates, whereas other species such as invertebrate only have one ERM gene [7]. ERM proteins function as general cross-linkers between plasma membrane proteins and the cortical actin cytoskeleton [8]. They can assemble multiprotein complexes at the membrane-cytoskeleton interface, thereby regulating numerous signal transduction pathways, and they also function as both upstream and downstream effectors of Rho GTPases. The lack of ERM function results in serious defects or mortality in vertebrates [9] and is lethal in invertebrate species [10,11].

Recent studies indicate that the regulation of cortical actin dynamics is only a part of the function of ERMs. Similarly to their major binding partner, actin and other cytoskeletal proteins [1], ERMs have been found to localize to the cell nucleus in numerous species [12-15]. In Drosophila melanogaster, the sole ERM representative, Moesin (Moe), has been shown to be a functional component of the nucleus by participating in mRNA export [16]. However, the in vivo importance of the nuclear localization and activity of ERMs is not known at present. Here, we demonstrate that the constant removal of Moe from the nucleus, with the help of a nuclear export signal (NES), results in abnormal development, sterility, and physiological problems in Drosophila. We also show that Moe plays role in the expression of 686 genes, among them heat-shock ( $h s p)$ genes. Our study provides the first direct evidence that the activity of the actin-binding ERM proteins in the cell nucleus is essential for the normal development and life of an organism.

\section{Results}

\section{Generation of the $m o e^{\text {NES }}$ mutant}

To obtain more insight into the nuclear activities of Drosophila Moe and to determine the biological relevance of this nuclear localization, we created mutant animals with decreased level of nuclear Moe. We fused the protein with a NES which ensures constant removal of Moe from the nucleus (Fig. 1A). We chose the evolutionarily highly conserved, chromosomal region maintenance 1 (CRM1)-recognized NES sequence motif of the cAMP-dependent protein kinase inhibitor alpha (PKIA) [17] and tested the effect of this NES-tag on the intracellular distribution of Moe in a pilot experiment. The nuclei of Drosophila larval salivary gland cells expressing GFP-labeled MoeNES protein from a transgene showed unambiguous decrease in the nuclear Moe level (Fig. 1B).

We then tagged the Moe protein in situ with the NES sequence using CRISPR-Cas9 technology [18]. In Drosophila, the moe gene is located on the X chromosome and with the use of alternative START sites and introns it encodes 13 different transcripts that give rise to seven different protein isoforms. We inserted the NES sequence in front of the STOP codon between the 14th exon and the $3^{\prime}$ UTR which are present in all 13 moe transcripts. For the selection of the mutants, the fluorescent dsRed expressing marker gene $(3 x P 3>$ dsRed) flanked by two loxP recombination sites was inserted into the last intron (Fig. 1C). From the embryos co-injected with the guide RNAs (gRNAs) and the donor construct, four independent mutant lines, hereafter referred to as $m o e^{\mathrm{NES}}$, were generated. Next, the dsRed marker gene was removed with the help of Cre recombinase, leaving a single loxP sequence in the last intron of the moe gene. Sequencing of the mRNA expressed from the moe $e^{\text {NES }}$ gene verified successful insertion of the NES sequence, the lack of the marker gene, and the normal excision of the last intron (data not shown).

We noticed that all the progenies of the females carrying at least one copy of the $m o e^{\mathrm{NES}}$ allele were sterile. The sterility was specific to mothers only; the progeny of hemizygous mutant males $\left(m o e^{\mathrm{NES}} / \mathrm{Y}\right)$ were fertile, indicating that the phenotype is due to a dominant maternal effect. Therefore, to maintain the $m o e^{\mathrm{NES}}$ allele for future experiments, mutant males were crossed to females carrying attached $\mathrm{X}$ chromosomes $(\mathrm{XX} / \mathrm{Y})$ to establish the stable moe ${ }^{\mathrm{NES}}$ stock. The attached $\mathrm{X}$ chromosome consists of two fulllength $\mathrm{X}$ chromosomes sharing a common centromere so that they are always inherited together. As a result, in crosses of $m o e^{\mathrm{NES}}$ males to females carrying an attached $\mathrm{X}$, male progenies inherit their moe $e^{\mathrm{NES}}$ bearing $\mathrm{X}$ from their father and their $\mathrm{Y}$ from their mother, while the females never carry the moe $e^{\mathrm{NES}}$ allele. In this way, the problem of maternal female sterility is eliminated. The $m o e^{\mathrm{NES}} / \mathrm{Y}$ mutant males were viable and fertile; however, to analyze females carrying at least one copy of the $m o e^{\mathrm{NES}}$ allele, $m o e^{\mathrm{NES}}$ males were crossed in every experiment to females carrying a wildtype (WT) X chromosome $\left(w^{1118}\right)$ or a null allele $\left(m o e^{\mathrm{G} 0415}\right)$ of moe. The resulting female progeny $\left(m o e^{\mathrm{NES}} /\right.$ moe $^{\mathrm{G} 0415}$, referred to as moe $e^{\mathrm{NES}}$ mutant in the text) expressing only the Moe ${ }^{\mathrm{NES}}$ protein were used in the assays (Fig. 1D) and compared throughout the experiments to control animals carrying either the moe 
A
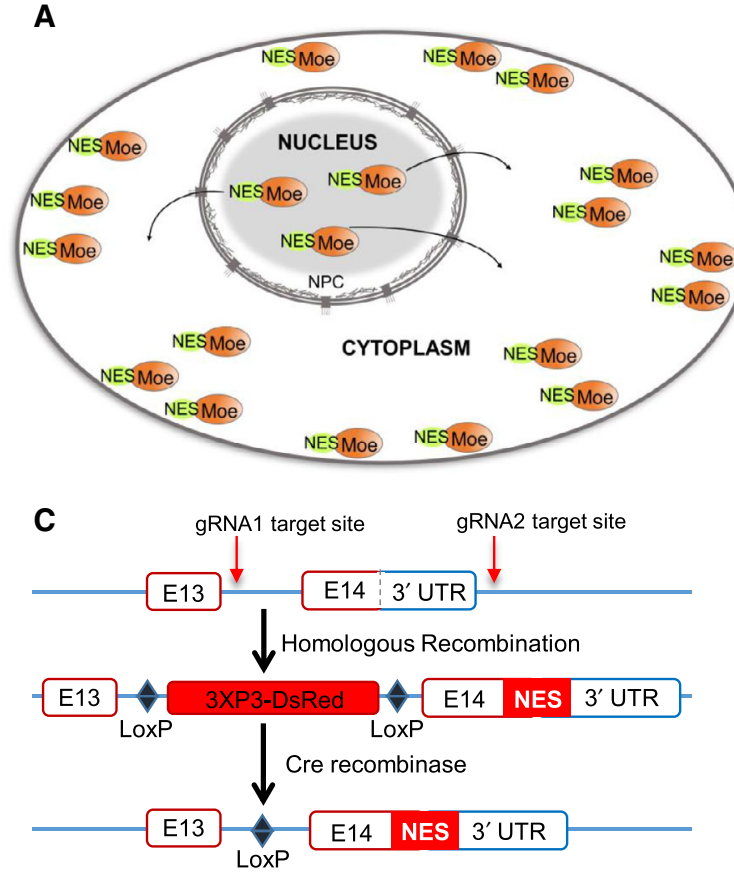

B Moe-GFP

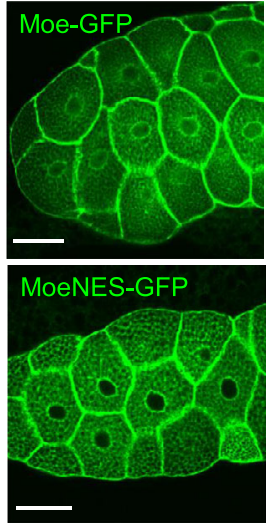

D

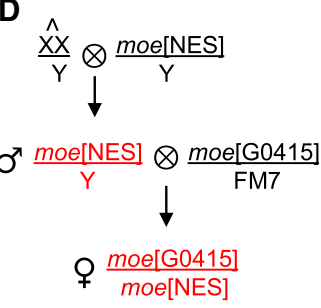

Fig. 1. Rationale of the experimental system. (A) NES ensures that Moe is constantly exported from the nucleus while its cytoplasmic functions remain intact. NPC: Nuclear pore complex. (B) Live images of larval salivary glands expressing Moe-GFP or MoeNES-GFP (green) demonstrate that MoeNES-GFP is not present in the nucleus. Representative images of salivary glands of eight larvae examined each genotype in one experiment. Scale bars, $50 \mu \mathrm{m}$. (C) In vivo tagging of the moe gene with NES signal using the CRISPR-Cas9 system. The NES sequence was inserted between the protein-coding sequence and the $3^{\prime} U T R$. (D) The moe ${ }^{\text {NES }}$ allele is maintained with the help of attached $X$ females. To generate moe ${ }^{\mathrm{NES}}$ mutant females (highlighted in red), the $m o e^{\mathrm{NES}} \mathrm{Y}$ mutant males (red) are crossed in all experiments with females carrying a moe null allele (moe $\left.e^{\mathrm{G0415}}\right)$

null $\left(m o e^{\mathrm{G} 0415} / w^{1118}\right)$ or the $m o e^{\mathrm{NES}}$ allele $\left(m o e^{\mathrm{NES}} /\right.$ $w^{1118}$ ) in heterozygous form.

\section{The cytoplasmic activity of the MoeNES protein is unaffected}

The complete loss of Moe functions is lethal to the fly. Null mutant animals die at the third larval stage $[19,20]$. In contrast, the moe $e^{\mathrm{NES}}$ mutant females and males were viable, suggesting that at least the main cytoplasmic functions of Moe are unaffected. In the next experiments, we aimed to confirm this notion.

In order to verify that the NES-tag does not inhibit the activation and activity of the Moe protein, salivary glands of $m o e^{\mathrm{NES}}$ mutant males and ovaries of $m o e^{\mathrm{NES}}$ mutant mothers were stained with an anti-phosphoERM antibody specifically recognizing the activated form of Moe [21]. The immunostaining revealed that the MoeNES protein is activated through phosphorylation and, similarly to the WT protein, it primarily localizes to the cell cortex (Fig. 2A). This demonstrates the F-actin-binding ability of MoeNES and suggests that its molecular cross-linking function is intact. The quantitation of the immunostainings showed that, in addition to localization, the nucleo-cytoplasmic distribution of P-Moe is normal in the moeNES mutant ovary (Fig. 2A'). The measurement of the levels of PMoe and Moe in the ovary by western blotting (Fig. 2A") suggested that the amount of P-Moe relative to total Moe amount in the mutant differs only by $10 \%$ from that of the control $(1.38$ and $1.52 \mathrm{Moe} / \mathrm{P}$ Moe ratio in the mutant and the control, respectively). The quantitation also revealed that the total amount of Moe is reduced by $30 \%$ in the $m o e^{\mathrm{NES}}$ mutant (142 837 vs. 99815 intensity values in the control and the mutant, respectively).

The female fly possesses two ovaries joined with a common oviduct, and around 16 ovarioles per ovary. The ovarioles are parallel strings of 6-7 progressively older egg chambers. The egg chamber is covered with a monolayer of somatic epithelial cells, the follicle cells. In the egg chambers, 15 polyploid nurse cells are connected to the oocyte and deposit a large amount of RNA and protein into it. During stage 10 of oogenesis, nurse cells produce unipolar, filopodia-like actin cables between their cell membrane and nucleus to 


\section{A}
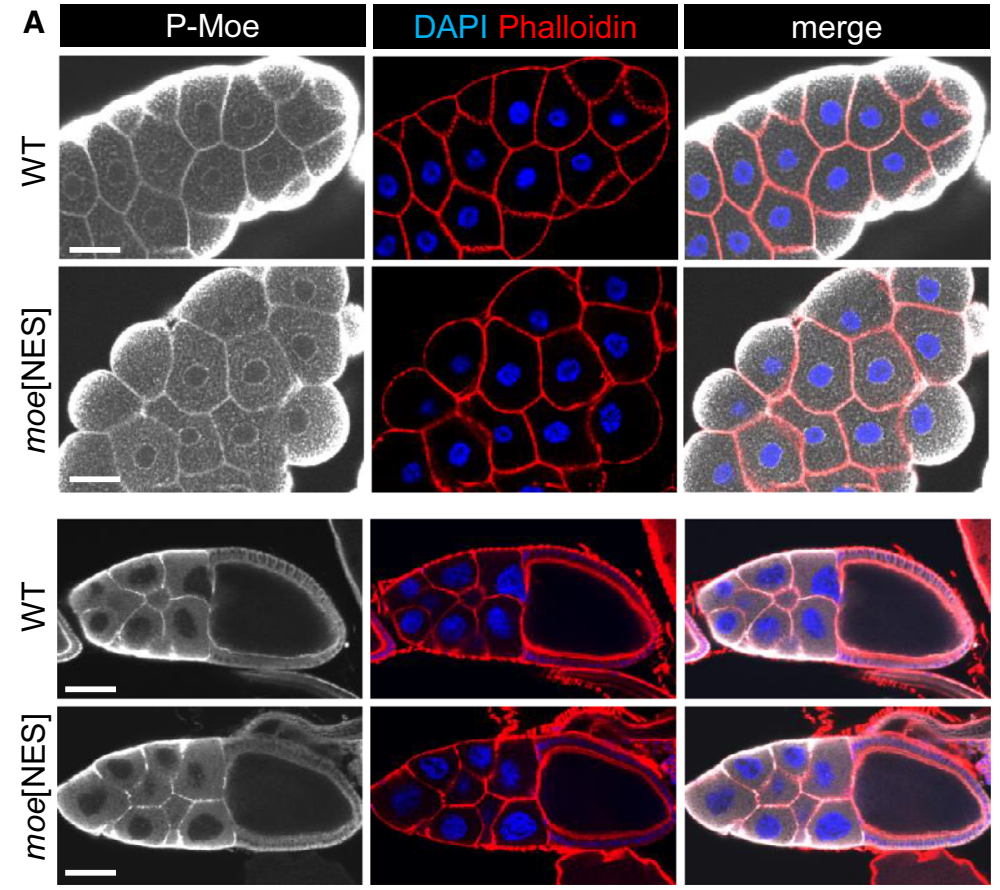

$A^{\prime}$

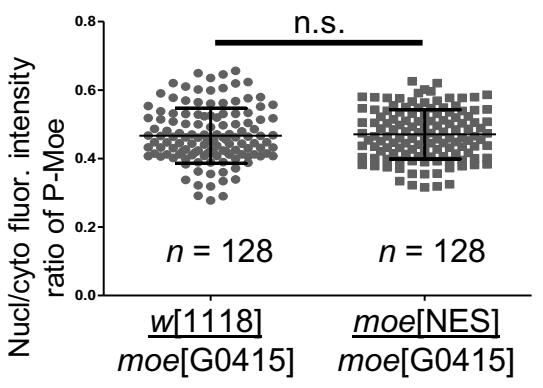

B
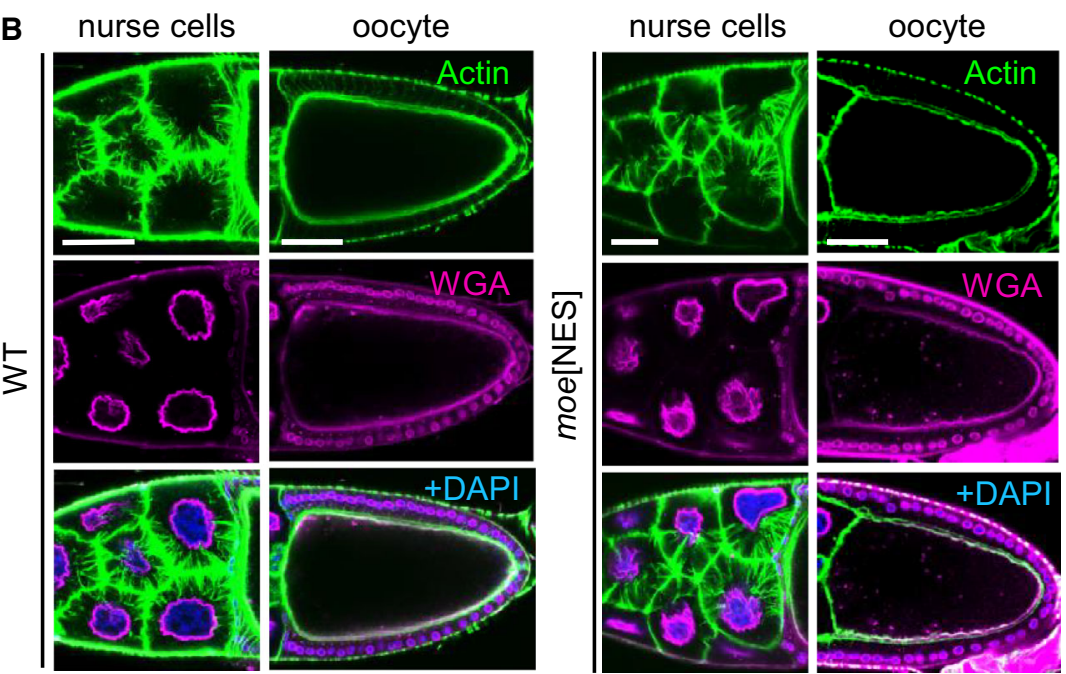

C
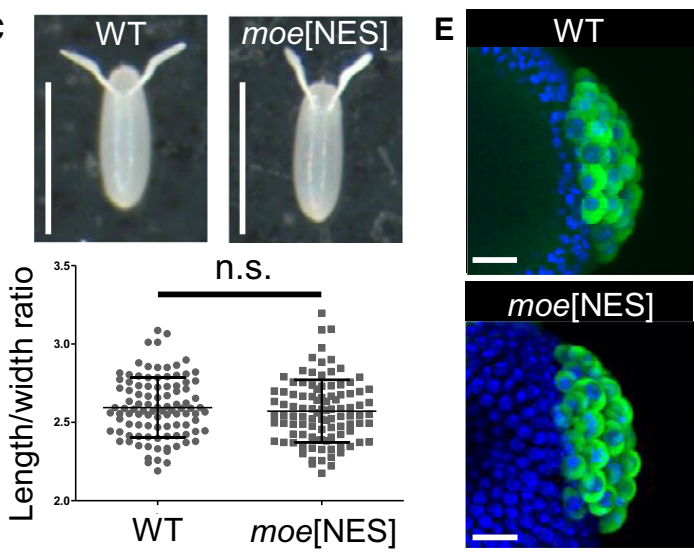

F
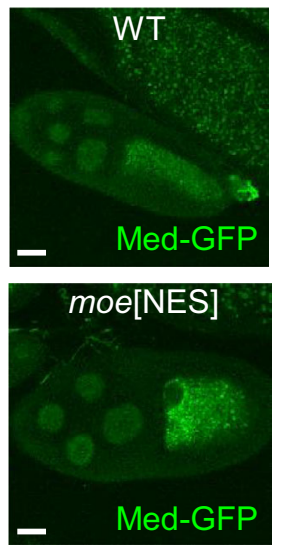
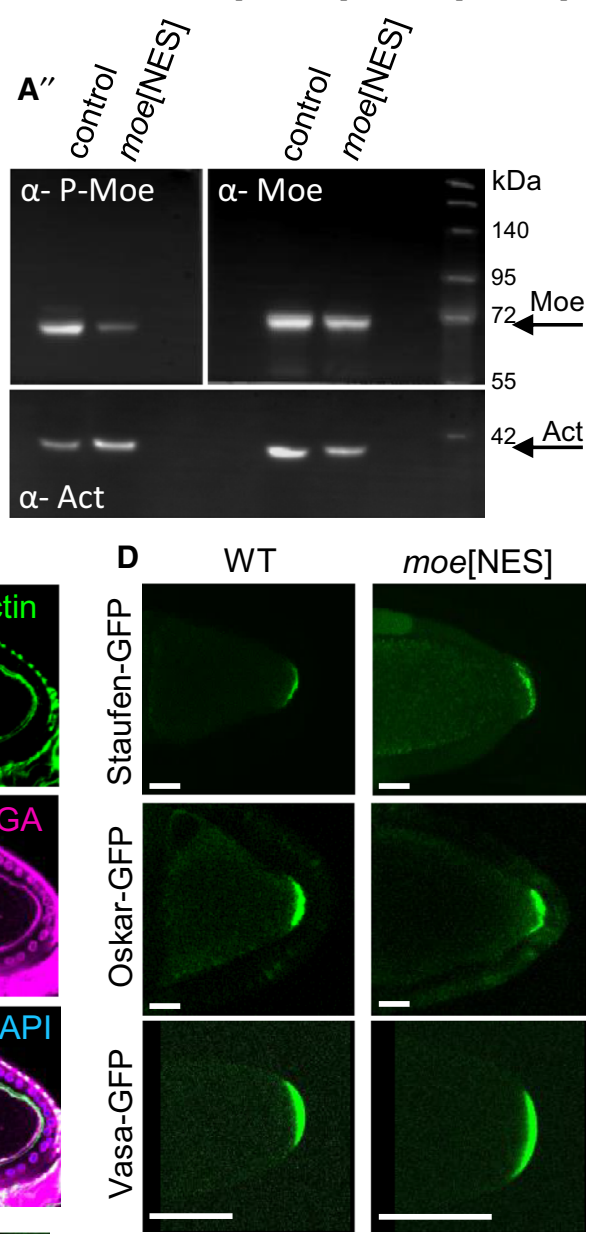

moe[NES]
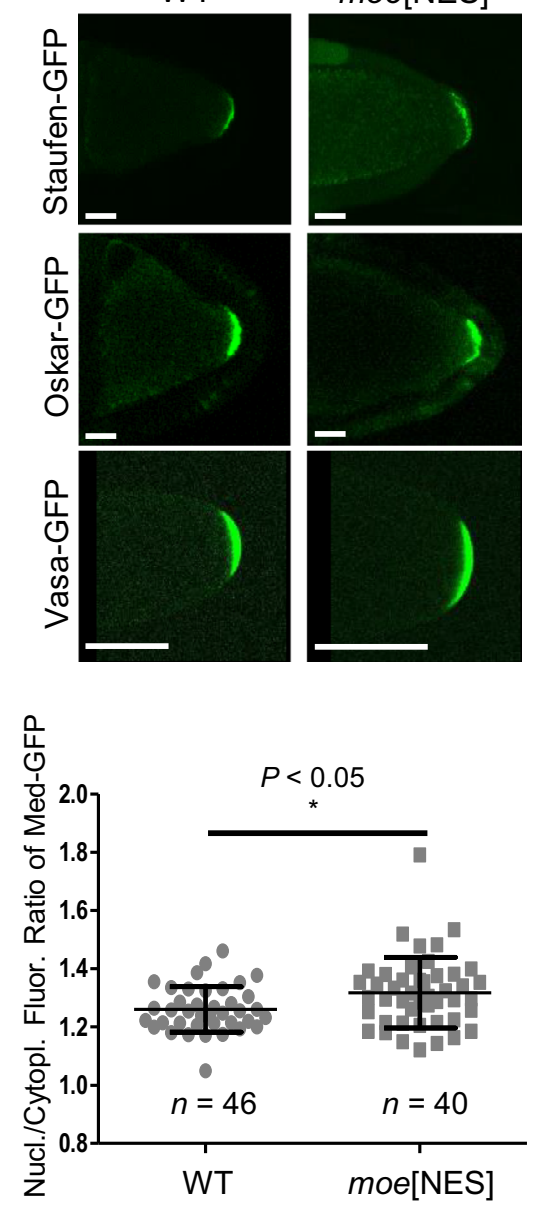
Fig. 2. The MoeNES protein functions normally in the cytoplasm. (A) Immunostaining for phospho-Moe (P-Moe, white) in the larval salivary gland (upper rows) and the ovary (bottom rows) reveals that the MoeNES protein is phosphorylated and localizes in the cytoplasm normally. Representative images of three independent experiments. $W^{1118}$ flies were used as WT. Scale bars, $50 \mu$ m. (A') Nuclear/cytoplasmic fluorescence intensity ratios in moe ${ }^{\mathrm{NES}}$ mutant and control ovaries of three independent experiments. Horizontal line represents mean fluorescent ratio $\pm S D$, ' $n$ ' is the number of cells examined, n.s.- -not significant (unpaired $t$-test). (A") Quantitation of the amount of Moe and P-Moe proteins in $m o e^{\mathrm{NES}}$ mutant $\left(\mathrm{moe}^{\mathrm{NES}} / \mathrm{moe}^{\mathrm{G} 0415}\right)$ and control $\left(m o e^{\mathrm{G0415}} / w^{1118}\right)$ ovaries with western blotting. Representative pictures of one of three independent experiments. Actin was used as loading control. Act-Actin. (B) The organization of the actin network and the position of the nuclei are intact in stage 10B egg chambers of $\mathrm{moe}^{\mathrm{NES}}\left(\mathrm{moe}^{\mathrm{NES}} / \mathrm{moe}^{\mathrm{G} 0415}\right.$ ) females. Phalloidin was used to stain for actin: green, WGA for membrane staining: magenta, DAPI: blue. $W^{1118}$ flies were used as WT. Representative images of at least 35 egg chambers examined in two independent experiments. Scale bars, $50 \mu \mathrm{m}$. (C) The eggs laid by moe ${ }^{\mathrm{NES}}$ mutant mothers have WT size and shape. $w^{1118}$ embryos were used as WT. Scale bars, $500 \mu \mathrm{m}$. Dot plot data represent the mean \pm SD of length/width ratio of eggs. n.s.not significant (unpaired t-test). 100 embryos were measured each genotype. (D) Immunostaining reveals that the localization of the maternal factors Staufen and Oskar (green) are normal in the oocytes of stage 9 egg chambers and that Vasa (green) localizes properly in the embryos of moe NES mutant females. Representative images of one of three independent experiments. Scale bars, $10 \mu \mathrm{m}$ (Staufen and Oskar) and $100 \mu \mathrm{m}$ (Vasa). (E) The number of pole cells at the posterior pole of the embryo laid by moe ${ }^{\text {NES }}$ mothers is normal as reflected by the immunostaining for Vasa (green) and DAPI (blue). $W^{1118}$ embryos were used as WT. Scale bars, $10 \mu$ m. Representative images of two independent experiments. (F) Medea localizes properly in moe NES mutant ovaries. Representative images of two independent experiments. The nuclear/cytoplasmic fluorescence ratio of the Medea-GFP signal reveals that the nuclear level of Medea (green) is unaffected in the ovaries of moe ${ }^{\text {NES }}$ females. $w^{1118}$ flies were used as WT. Stage 8 egg chambers are shown. 20 egg chambers of 10 females were analyzed of each genotype. ' $n$ ' indicates the total number of nurse cells measured. Error bars $=S D, * P<0.05$, unpaired t-test. Scale bars, $50 \mu \mathrm{m}$.

prevent the nuclei from moving and to block the squeezing of cytoplasm into the oocyte [22]. Moe is required for the formation of these tethering actin cables in the ovary and anchors the cortical actin network to the cell membrane in the developing oocyte. Therefore, the impairment of Moe function leads to the disorganization of the microfilament network [21] and the detachment of cortical actin from the cell membrane [19]. Visualization of the actin cytoskeleton and the membranes at stage 10 of oogenesis in ovaries of control $\left(m o e^{\mathrm{G} 0415} / w^{1118}\right)$ and $m o e^{\mathrm{NES}}$ mutant females demonstrated that the intracellular position of nurse cell nuclei and the actin network are normal in the mutant (Fig. 2B). The perturbation of nurse cell filamentous actin cable organization or their integration with the cortical actin network blocks nurse cell dumping which in turn results in reduced embryonic size [23]. The regular size and shape of the embryos laid by $m o e^{\mathrm{NES}}$ mutant mothers confirmed further that the cytoskeletal functions of Moe are unaffected (Fig. 2C).

The intact cytoskeletal network of the oocyte also ensures the correct localization of maternal factors within the cytoplasm, which is necessary for the formation of germ cell precursors at the posterior pole of the developing embryo. Moe plays essential role in this process, as demonstrated previously by the mislocalization of maternal factors Oskar, Staufen, and Vasa, and significantly reduced number of pole cells $[19,21]$ in the embryos of moe $e^{\mathrm{NES}}$ mutant mothers. However, we found that in the eggs laid by moe $e^{\mathrm{NES}}$ mutant females, the maternal factors Oskar, Staufen, and Vasa all localize properly at the posterior pole of the oocyte or the early embryo (Fig. 2D), and at the cellularization phase, the number of embryonic pole cells is normal also (Fig. 2E), providing additional evidence that the cytoplasmic functions of MoeNES are normal.

The MoeNES protein is exported from the nucleus by the CRM1 (XPO1) export factor which recognizes the NES motif. In the next set of experiments, we investigated whether the expression of the MoeNES protein alters the normal activity of CRM1, for example, by overloading the CRM1-mediated nuclear export pathway. We thus compared the ratio of nuclear and cytoplasmic fluorescent intensity values of the GFP-tagged Medea (SMAD4) protein, a known cargo of the CRM1 exportin [24,25], in the nurse cells of WT and mutant ovaries (Fig. 2F). We expected the nuclear accumulation of Med-GFP if the export is overloaded, but the protein exhibited very similar nuclear-cytoplasmic distribution in normal and mutant nurse cells. This confirms that the CRM1 nuclear export pathway functions normally in the moe $e^{\mathrm{NES}}$ mutant and that reduced CRM1 pathway activity cannot be responsible for the observed phenotypes.

\section{Lack of nuclear Moesin causes diverse phenotypes}

The moe $e^{\text {NES }}$ mutant males and females were viable and fertile; however, they exhibited numerous phenotypes (Fig. 3A). These defects, such as slow development, decreased lifespan, egg production and climbing ability, as well as male genitalia rotation, were of 
A

Fig. 3. Phenotypes of moe $e^{\mathrm{NES}}$ mutant animals. (A) Both sexes of moe $\mathrm{NES}^{\mathrm{N}}$ mutant parents $(\mathrm{P})$ exhibited slow development, decreased lifespan, heat stress sensitivity, and lethality. The decreased climbing ability and genitalia rotation are manifested only in moe ${ }^{\text {NES }}$ males, while moe ${ }^{\text {NES }}$ mutant females show grandchildless phenotype. The F1 offsprings of moe ${ }^{\mathrm{NES}}$ mothers are $100 \%$ sterile and have various

developmental problems. (B) Rotated male genitalia in moe $^{\mathrm{NES}} / \mathrm{Y}$ parents $(32.4 \%$ of 145 males examined). (C) All the F1 offsprings of moe NES mutant mothers have

rudimentary and nonfunctional genitalia (100\% of 82 flies). (D) Developmental problems of F1 progeny include tergit development and pigmentation problems $(10.7 \%)$, extra bristles (1.2\%), absence of genitalia (5.8\%), notched wing margin and reduced or missing cross-veins $(4.9 \%)$, darker and rough eye (100\%). Three hundred and fifty flies were analyzed. Defects are circled or marked by red arrows. WT $\left(w^{1118}\right)$, A5-A6: abdominal segments 5 and 6, ACV: anterior cross-vein, PCV: posterior cross-vein.
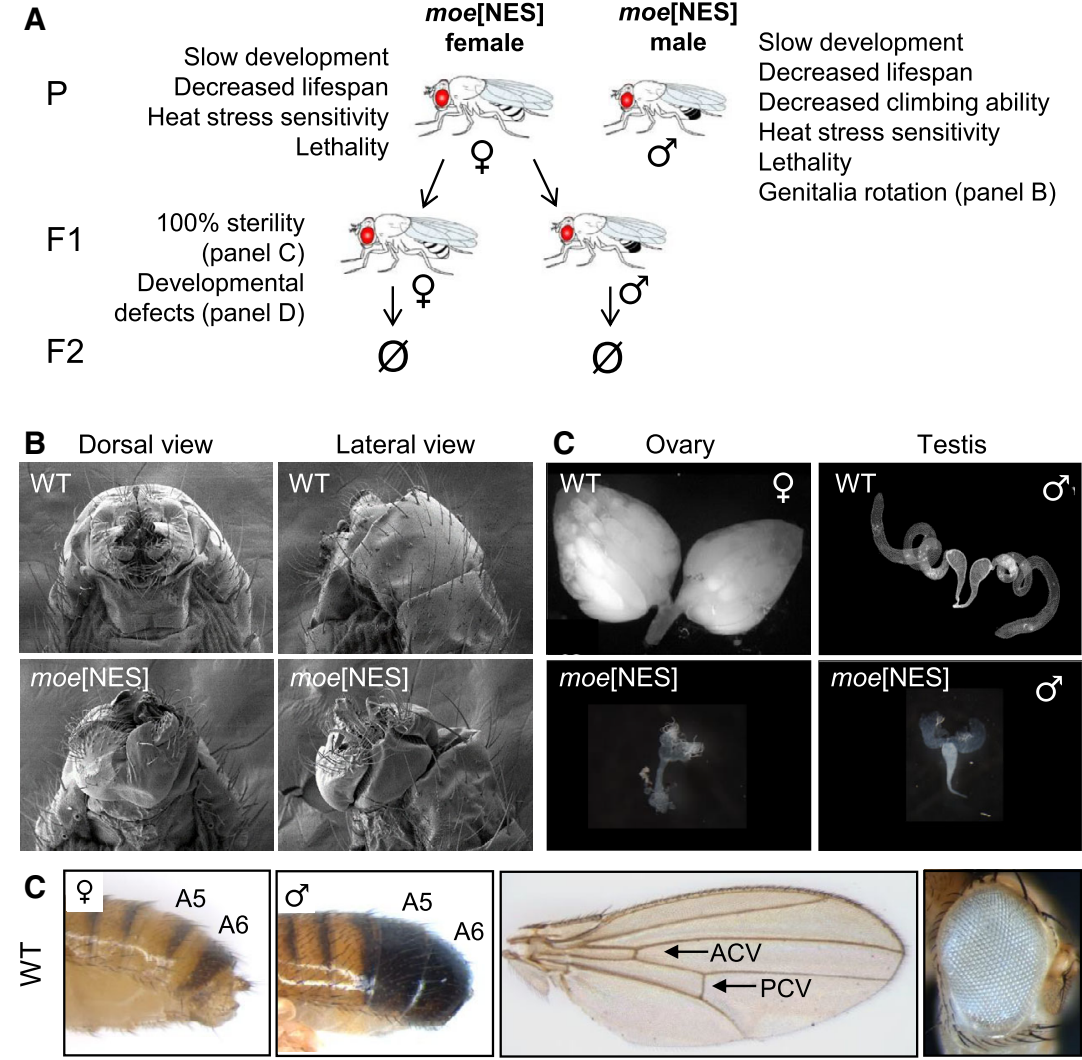

$\sigma^{\top}$
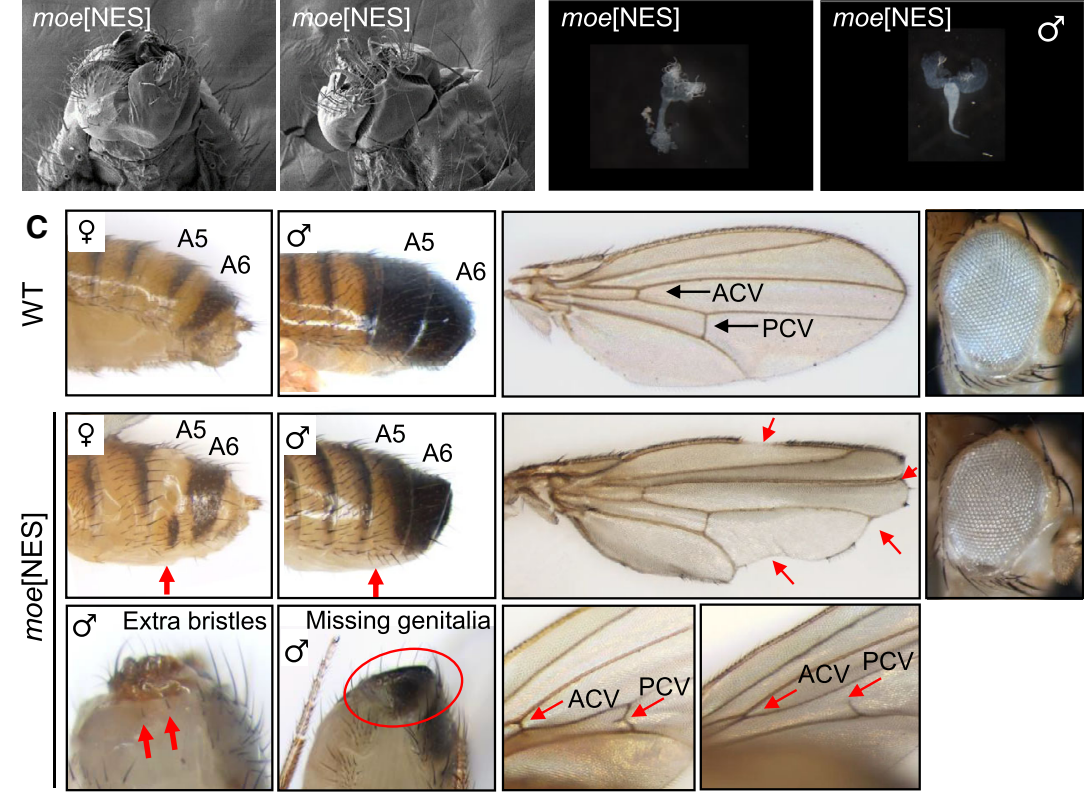

zygotic origin. The ratio of moe $e^{\mathrm{NES}}$ mutant males with genitalia rotation (Fig. 3B) was $32.4 \%$ (out of 145 animals), and the frequency of this phenotype decreased to $12.3 \%$ (out of 171 animals) when the males were bearing a $\mathrm{Y}$ chromosome that contained one copy of the WT moe gene (Dp(1:Y)619). This suggests that an extra copy of the moe gene can rescue genitalia rotation by about $60 \%$ and that the phenotype is due to the loss of Moe function.

In contrast to the zygotic phenotypes, maternal-effect phenotypes appear in the F1 descendants of $m o e^{\mathrm{NES}}$ females, independently from the genotype of the progeny. The maternal phenotypes primarily affected the posterior part of the animals and caused rudimentary genitalia and, as a consequence, sterility (Fig. 3A). The maternal-effect sterility of the F1 descendants was due to the undeveloped genitalia both in males and in females (Fig. 3C). The visible phenotypes in the $\mathrm{F} 1$ progeny of moe $e^{\mathrm{NES}}$ mutant mothers were defects in tergite development and pigmentation, extra hairs, wing malformations, and dark and rough eye (Fig. 3D).

The $m o e^{\mathrm{NES}}$ mutant stock was weak, and thus, we analyzed the viability of the animals at every main developmental stage. Out of 100 eggs laid by the moe $e^{\mathrm{NES}}$ mutant mothers, only $30 \%$ hatched, in contrast to the control $\left(m o e^{\mathrm{G} 0415} / w^{1118}\right)$ where $98 \%$ of the embryos developed to larval stage. In addition, embryonic viability further decreased with the aging of the mutant mothers $(0 \%$ in the mutant vs. $92 \%$ in the control). The phenotype, especially at younger age, was partially rescued by a WT moe allele $\left(m o e^{\mathrm{NES}} /\right.$ 
A

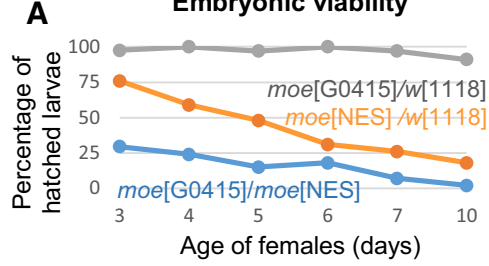

Pupal viabilty
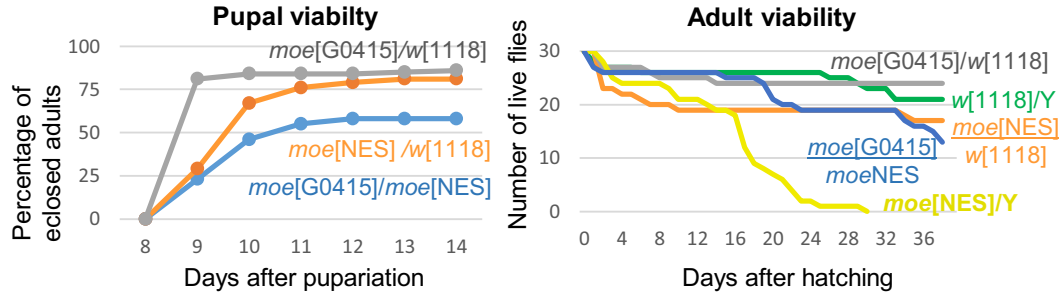

B

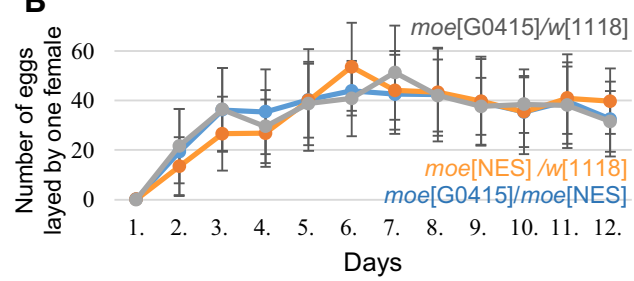

Fertility test
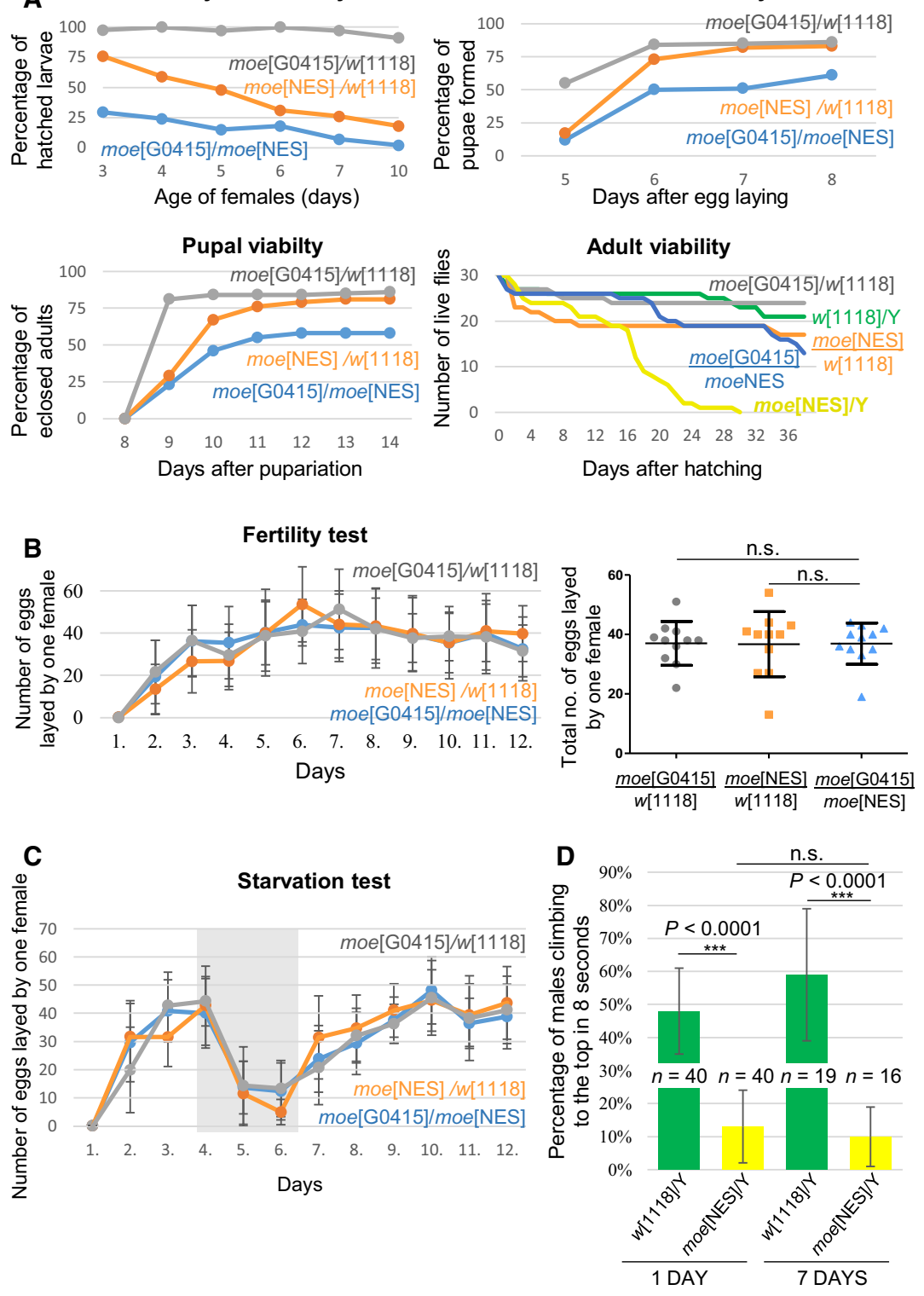

Adult viability

Days after hatching
Larval viability

Fig. 4. $m o e^{\mathrm{NES}}$ mutants have physiological problems. (A) moe ${ }^{\mathrm{NES}}$ mutant animals show increased lethality at every developmental stage. Decreased lifespan at $25^{\circ} \mathrm{C}$ is observed only in $m o e^{\mathrm{NES}} / \mathrm{Y}$ males. (B) The fertility test shows that there is no difference between the moe $e^{\mathrm{NES}} / \mathrm{moe}^{\mathrm{G} 0415}$ and control females in the number of laid eggs. The egg yield is normal during a $12-$ day period as shown in the graph $(n=16$, error bars $=\mathrm{SD}$ ). The total number of eggs laid by one female is also normal (Dot plot). Error bars $=S D, n=11$, n.s. - not significant (unpaired $t$-test). (C) The response of moe ${ }^{\mathrm{NES}}$ females to starvation is normal. Data represent the mean $\pm S D$ of the number of eggs laid by one female. 30 females were tested of each genotype. (D) $m o e^{\mathrm{NES}} / \mathrm{Y}$ mutant males perform poorly in the climbing test: only $13 \%$ of them is able to climb the full distance in $8 \mathrm{~s}$, in contrast to $48 \%$ of the WT. The ability of climbing did not decrease significantly with age. Data show quantitative evaluation (mean $\pm \mathrm{SD}$ ) of three independent experiments. n.s.not significant, $* * * P<0.0001$ (unpaired $t$ test), ' $n$ ' is the number of flies examined. In all panels, genotypes are indicated by the following colors: gray-moe $e^{\mathrm{G} 0415} / w^{1118}$, orange-moe $e^{\mathrm{NES}} / w^{1118}$, blue-moe $e^{\mathrm{G} 0415} /$ $m o e^{\mathrm{NES}}$, green $-w^{1118} / \mathrm{Y}$, yellow-moe ${ }^{\mathrm{NES}} \mathrm{Y}$. $w^{1118}$ ) (Fig. 4A). DAPI staining of early embryos demonstrated that, similarly to the progeny of control females, $100 \%$ of the eggs laid by moe $e^{\text {NES }}$ mutant mothers $(n=100)$ pass the syncytial blastoderm stage, indicating that lack of fertilization does not contribute to embryonic lethality (not shown). After the embryonic stage, $30 \%$ lethality was observed at both larval and pupal stages of moe $e^{\mathrm{NES}}$ mutants. The viability at these stages was fully rescued by one copy of the WT moe gene $\left(m o e^{\mathrm{NES}} / w^{1118}\right)$, indicating that the lethality during development is caused by the loss of Moe function. These data also demonstrated that the development of mutant animals is slower compared with the control. The viability experiments revealed a shortened adult life span only in the case of $m o e^{\mathrm{NES}} / \mathrm{Y}$ mutant males. In these males, life span was found to be decreased by $\sim 50 \%$ compared with mutant females or control males. Since these flies displayed a reduced hatching rate, we analyzed the fecundity of $m o e^{\mathrm{NES}}$ mutant females. They exhibited normal egg production, the daily egg yield as well as the total number of eggs laid by one female during the examined 12-day period were almost identical to those of the control females (Fig. 4B).

In the next set of experiments, we inspected the possibility that low energy homeostasis is contributing to the phenotype. We measured the egg production of mutant and control females during starvation. Two 
days of food deprivation resulted in a dramatic decrease in egg production in both groups, followed by a rapid return to normal levels. The kinetics of egg yield changes were the same in both groups (Fig. 4C), indicating that moe $e^{\text {NES }}$ mutants show normal physiological reactions to temporary food deprivation and suggesting that altered energy homeostasis is not responsible for reduced life span.

During the experiments, we also noticed that mutant flies move more slowly than the control animals; therefore, we tested the basic locomotor behavior of the mutant (Fig. 4D). For the investigation of locomotor activity, we performed a negative geotaxis assay and found that mutant males move significantly more slowly than WT males, with only $13 \%$ of them climbing to the top of the vial during $8 \mathrm{~s}$, compared to $48 \%$ in case of the control animals. In contrast, the locomotor activity of mutant females was normal (not shown). We also conducted the test on 1-week-old males, but the difference between the mutant and the control flies was about the same (59\% vs. $10 \%$ in WT and mutant flies, respectively) (Fig. 4D).

\section{Transcriptome analysis reveals altered gene expression in moe $^{\mathrm{NES}}$ mutants}

Because the progeny of mutant females exhibit various maternal-effect phenotypes (reduced viability, developmental defects, sterility), and the ovary is the place of very extensive gene expression, we performed mRNASeq analysis on ovaries of $m o e^{\mathrm{NES}} / \mathrm{moe}^{\mathrm{G} 0415}$ mutant females, and compared their transcriptomes with $m o e^{\mathrm{NES}} /+$ and $m o e^{\mathrm{G} 0415} /+$ controls (Fig. 5). Principal component analysis (PCA) of the mRNA-Seq datasets showed $24 \%$ total variance in the first principal component (PC1) and clearly separated the mutant animals from the two controls (Fig. 5A), indicating significant differences between the transcriptomes of WT $\left(m o e^{\mathrm{G} 0415} / w^{1118}\right)$ and mutant samples. Compared to
WT $\left(m o e^{\mathrm{G} 0415} / w^{1118}\right)$, approximately the same number of differentially expressed genes were upregulated $(n=371)$ or downregulated $(n=315)$ in the mutant (Fig. 5B).

To gain more biologic insight, we performed Gene Ontology (GO) term analysis on statistically significant genes [twofold $\left(\log _{2}(\mathrm{FC}) \geq 1\right.$ and adjusted $P$-value of $<0.05$ for upregulated genes, and $\log _{2}(\mathrm{FC}) \leq-1$ and adjusted $P$-value of $<0.05$ for downregulated genes] differentially expressed between the genotypes $m o e^{\mathrm{G} 0415} / w^{1118}, m o e^{\mathrm{NES}} / w^{1118}$, and $m o e^{\mathrm{NES}} / m_{0} e^{\mathrm{G} 0415}$ by subjecting the data (summarized in Table S1) to the GO database of Flybase (https://flybase.org/). We observed that major transcriptional changes in upregulated genes related to cellular component terms involve the cell membrane and extracellular matrix, while in the case of downregulated genes, nuclear and nuclear + cytoplasmic terms were affected (Fig. 5B). The top biological process terms in both up- and downregulated gene groups are related to 'developmental process', 'response to stimuli', and 'transcription' (Fig. 5C). In addition, we found the GO terms 'chromosome organization' and 'cell cycle' in downregulated genes and 'signal transduction' and 'protein metabolic process' in the case of upregulated genes. These GO terms cover $68.3 \%$ of the downregulated genes and $51.5 \%$ of the upregulated genes.

Among the upregulated genes, three key regulators of development, vasa, Notch, and $d p p$, showed significant changes in gene expression activity (Fig. 5D), which may contribute to the developmental defects and sterility of moe NES mutants. Genes that might account for normal locomotor activity were also identified in the upregulated group (Fig. 5E), suggesting that abnormal gene expression may be responsible for the phenotype in adult males. These results show that the biological processes behind the phenotypes are impaired by gene programs that are directly affected in moe $e^{\mathrm{NES}}$ mutants.

Fig. 5. Altered gene expression activity in moe ${ }^{\mathrm{NES}}$ mutants. (A) PCA of the transcriptome of two independent replicates of the two control and the mutant ovaries. $X$ and $Y$ axes show principal component 1 and principal component 2 variance that explain $24 \%$ and $16 \%$ of the total variance, respectively. (B) From the data analysis, 12965 genes were identified; 371 genes had higher and 315 genes had lower transcript levels than the control groups. Based on the grouping of cellular components, the downregulated genes are predominantly nuclear (39\% only nuclear and 13\% nuclear and cytoplasmic), while the upregulated genes have the same nuclear and cytoplasmic ratio as the total gene group. (C) Biological function analysis. Three biological functions-developmental process, response to stimuli, and transcription-involve most of the genes that show increased or decreased transcript level. (D) Posterior developmental malformations in the mutants could be due to the change in the transcription level of maternal developmental factors. The transcript levels of the dpp and Notch genes, which play key roles in the Decapentaplegic (Dpp) signaling pathway, were significantly increased, while a small increase in the transcript level of vasa was detected. Error bar represents minimum and maximum values. Horizontal line $=$ mean of normalized gene expression values. (E) Six genes (bt, Mhc, Mlc1, Mlc2, Neurochondrin, and up) were identified with increased expression in the mutant (green) which can account for decreased climbing activity. Error bar represents minimum and maximum values. Horizontal line $=$ mean of normalized gene expression values. 


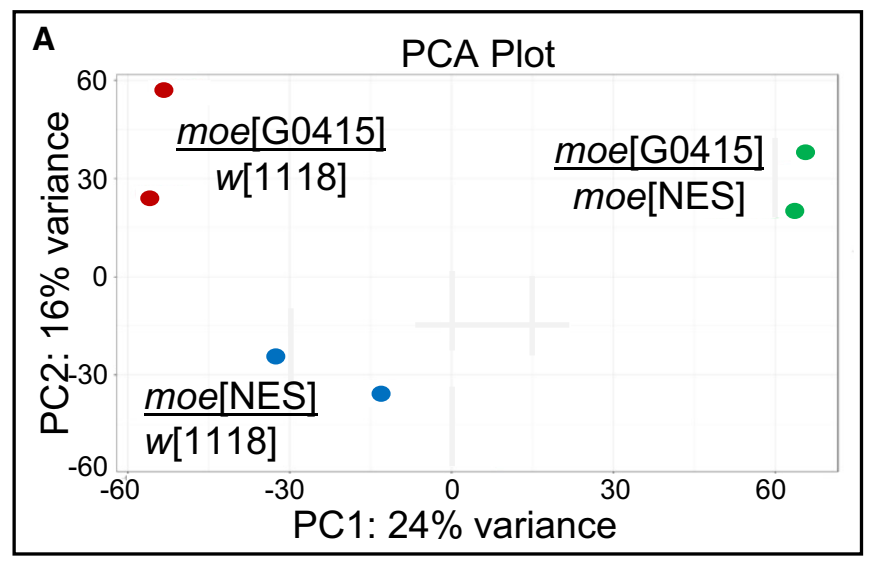

371 GENES UP-REGULATED

Without goup

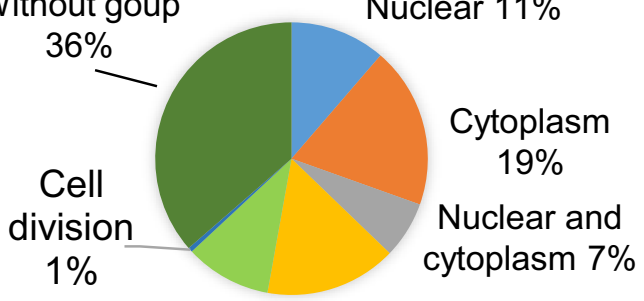

Extracellular space Membrane $10 \%$ associated $16 \%$
B $\quad 12,965$ GENES ANALYZED

Nuclear

Without group $13 \%$ $44 \%$<smiles>C1CCCC1</smiles>
space $5 \%$

Cytoplasm $22 \%$

Nuclear and cytoplasm $7 \%$ $9 \%$

\section{GENES DOWN-REGULATED}

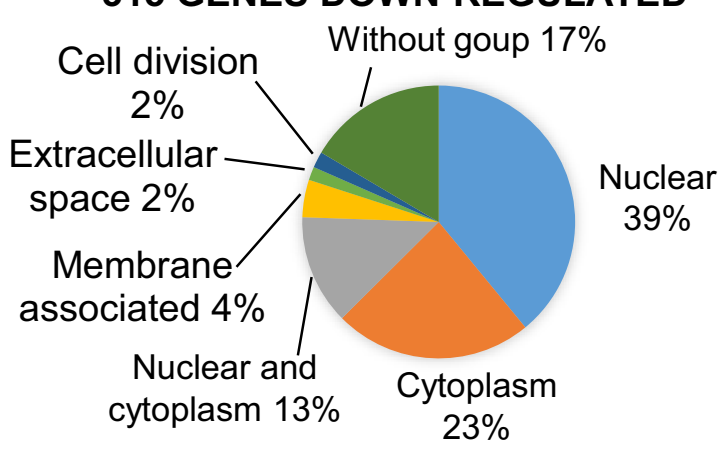

C UPREGULATED GENES

Developmental process
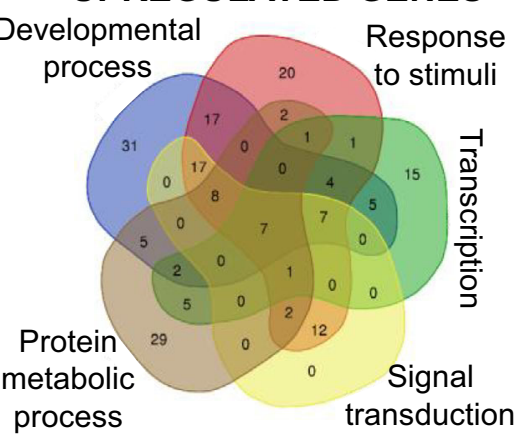

DOWNREGULATED GENES

Developmental process

Response

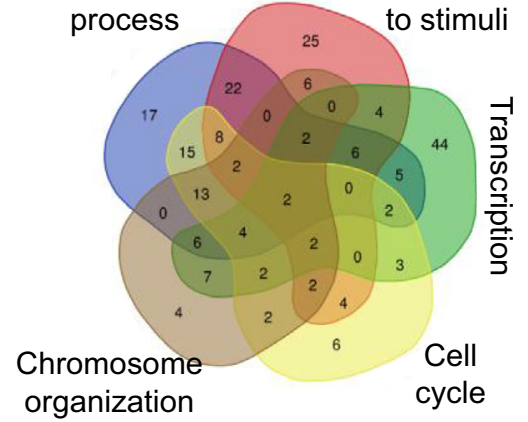

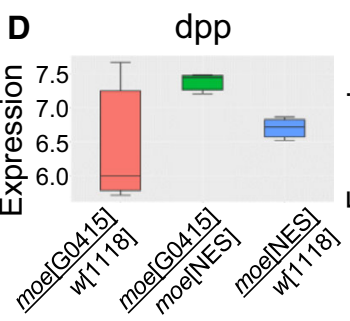

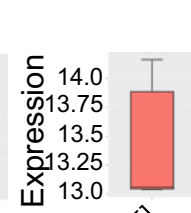

Vasa

Notch
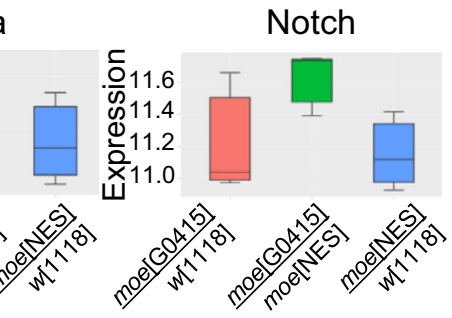

$E$

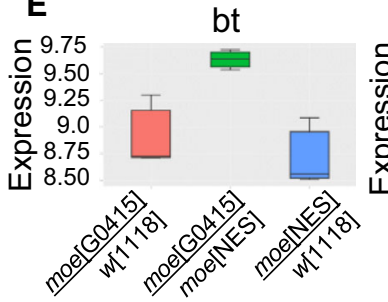

Mlc2

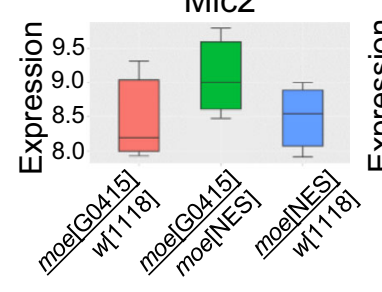

Mlc1

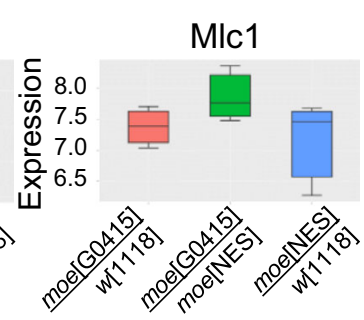

Mhc
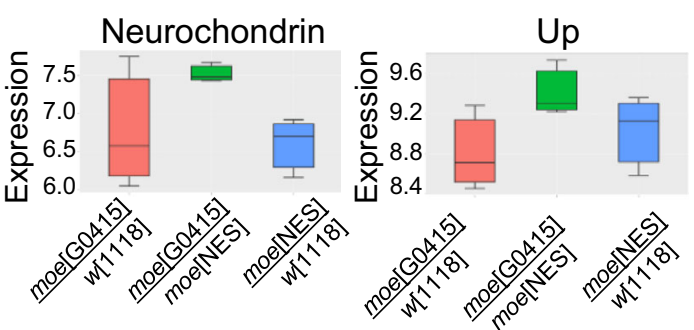
A

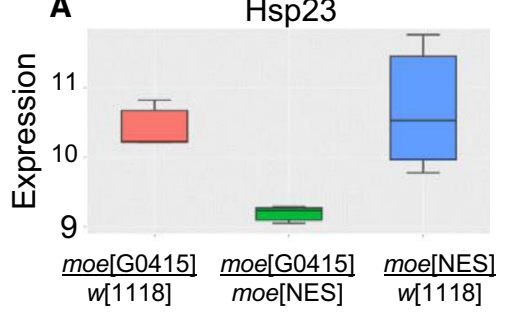

Hsp70Aa

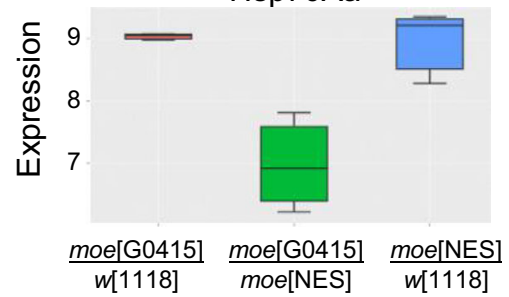

Hsp26

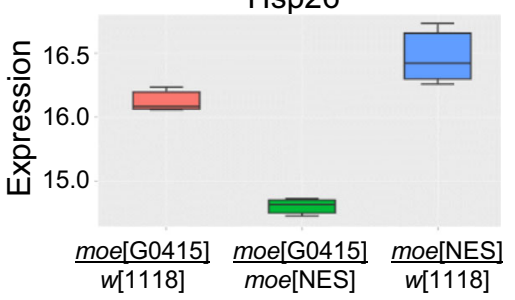

Hsp70Ab

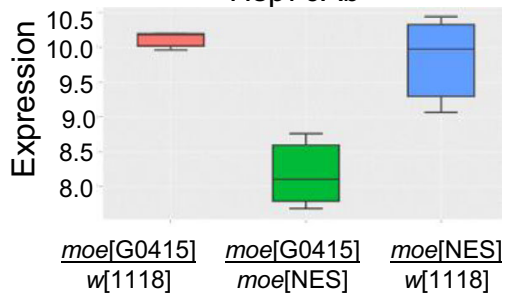

Hsp68

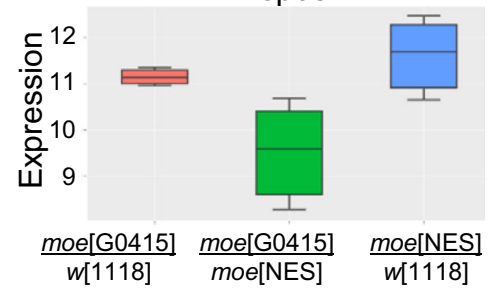

Hsp70Ba

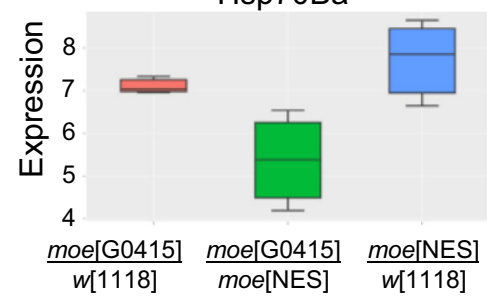

B

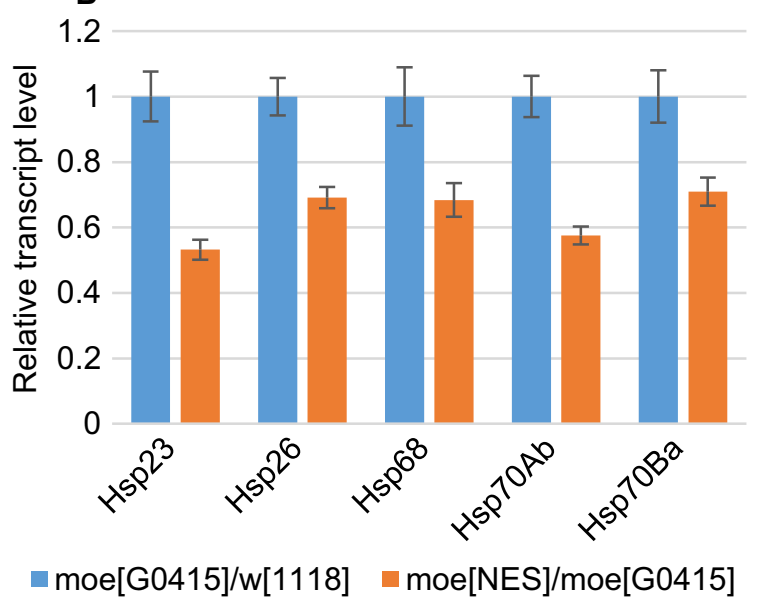

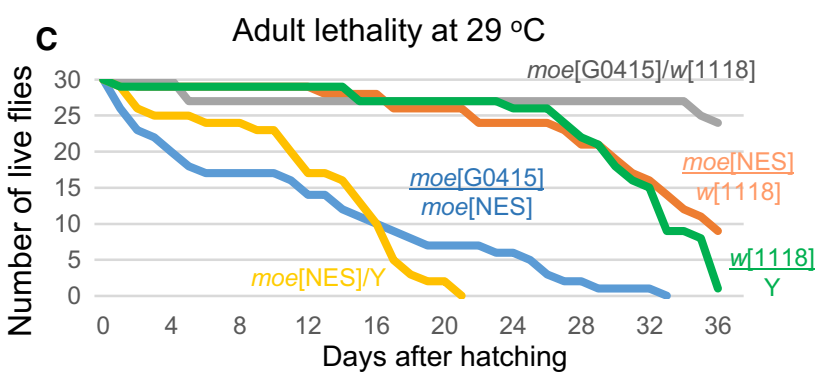

Adult lethality at $18^{\circ} \mathrm{C}$

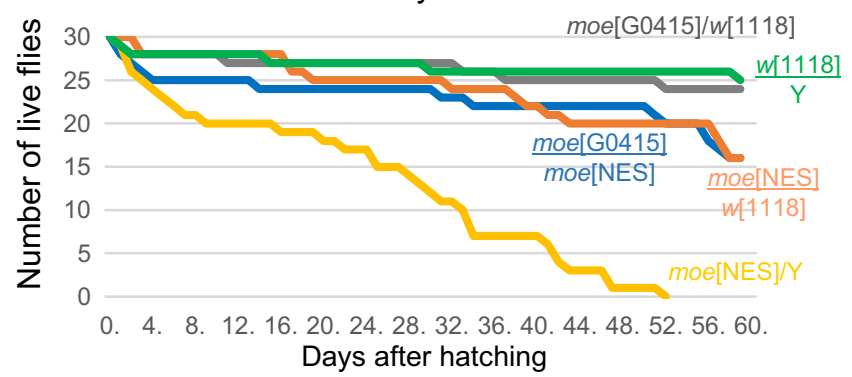

Fig. 6. Nuclear Moe is required for heat-shock gene expression. (A) The expression of six hsp genes is decreased in the moe ${ }^{\mathrm{NES}}$ mutant flies (green). Error bar represents minimum and maximum values. Horizontal line = mean of normalized gene expression values. (B) RTGPCR experiment on heat-shocked animals further demonstrates that nuclear Moe is required for hsp transcription. Data represent the mean $\pm \mathrm{SD}$ of relative transcript levels measured in ovaries from five females of each genotype. (C) Both sexes of moe $e^{\mathrm{NES}}$ mutant show decreased viability at $29^{\circ} \mathrm{C}$. Only mutant males exhibit shortened life span at $18{ }^{\circ} \mathrm{C}$. gray $-m o e^{\mathrm{G} 0415} / w^{1118}$, orange - moe $e^{\mathrm{NES}} / w^{1118}$, blue $m o e^{\mathrm{G} 0415} / \mathrm{moe} e^{\mathrm{NES}}$, green $-w^{1118} / \mathrm{Y}$, yellow $-m o e^{\mathrm{NES}} / \mathrm{Y}$.

\section{Nuclear Moesin is required for the transcription of heat-shock genes}

We noticed during the transcriptomic experiments that among the best hits in genes with downregulated expression in moe $e^{\mathrm{NES}}$ mutant, six genes encoded heat shock-inducible protein chaperons ( $h \mathrm{sp} 70 \mathrm{Aa}$, hsp $70 \mathrm{Ab}$, hsp70Ba, hsp68, hsp26, and hsp23) (Fig. 6A). The decrease in $h s p$ gene activity was rescued with one copy of WT moe allele, strongly suggesting that the nuclear activity of Moe is necessary for the normal expression of these genes. RT-qPCR on heat-shocked mutant animals reinforced the idea that nuclear Moe participates in the transcription of $h s p$ genes (Fig. 6B). In addition to thermotolerance [26,27], proper 
functioning of Drosophila $h s p$ genes is necessary for normal life span [28], fertility and germ cell maintenance $[29,30]$, locomotor activity [31,32], and developmental timing [27]. Since all these processes are affected in $m o e^{\text {NES }}$ animals, we performed additional experiments to confirm the involvement of Moe in $h s p$ gene activity.

The most obvious sign for hsp malfunctioning is heat intolerance; therefore, we first analyzed the viability of the mutants at elevated temperature. At $25^{\circ} \mathrm{C}$, only mutant males showed shortened life span (Fig. 4A), but at $29^{\circ} \mathrm{C}$, both sexes displayed a significant decrease in life span (Fig. 6C). The phenotype was fully rescued by one copy of the WT moe gene, indicating that loss of Moe function is responsible for the decreased heat tolerance. To further demonstrate that heat intolerance causes reduced viability at high temperature in males and females, we also performed the experiment at $18{ }^{\circ} \mathrm{C}$. The result was similar to the data obtained at $25^{\circ} \mathrm{C}$, only mutant males exhibited shortened life span; however, at this temperature, the mutant males lived much longer (51 vs. 21 days) than at $29^{\circ} \mathrm{C}$ (Fig. 6C). We also investigated whether the observed heat stress intolerance affects the negative geotaxis behavior of the mutants. The $35 \%$ decrease in the climbing ability of $m o e^{\mathrm{NES}}$ animals at $25^{\circ} \mathrm{C}$ (Fig. 4D) did not change significantly at higher temperature (data not shown), indicating that heat stress does not affect climbing activity. This in turn suggests that impaired $h s p$ expression does not contribute to locomotor problems of the mutant.

In our previous work, we discovered that upon heat shock the amount of Moe significantly increases in the nucleus [16]. Therefore, we tested if the NES-tagged Moe isoform can also localize to the nucleus at high levels, by ectopically expressing GFP-labeled Moe and Moe NES proteins in the larval salivary gland cells (Fig. 7A). In contrast to WT Moe, MoeNES-GFP did not accumulate in the nuclei after heat shock, demonstrating that the NES-tag inhibits the increase in Moe level in the nucleus. The immunostaining of WT and $m o e^{\text {NES }}$ larvae with an anti-Moe antibody revealed that, although the endogenous MoeNES protein is not completely eliminated from the nucleus, upon heat shock it fails to accumulate in the nucleus (Fig. 7B).

Moe has been shown previously to localize to the heat-shock puffs at cytological locations $87 \mathrm{~A}$ and $87 \mathrm{C}$ [16]. To further confirm that Moe is responsible for the regulation of heat-shock gene transcription, we tested whether WT Moe localizes to the actively transcribing heat-shock genes identified in the transcriptome experiment. The activation of these genes by heat stress leads to the formation of chromosome puffs, which are special euchromatic regions of the polytene chromosomes with extremely high levels of transcription. We found that Moe accumulated in high levels to the heat-shock puff sites, as demonstrated by the immunostaining of chromosome preparations from salivary gland cells (Fig. 7C), providing additional evidence that Moe is involved in the transcription of $h s p$ genes. Because in the cytoplasm Moe is organizing cytoskeletal structures, we wanted to exclude the possibility that Moe is needed for the formation of the puff structure. For this aim, we induced transcription with heat shock at a specific chromosomal location without the formation of the puff structure and stained for Moe. The animals used in this experiment carried a heat shock-inducible transgene at cytological location $86 \mathrm{E}$ in heterozygous form (Fig. 7D). The homologous chromosomes in the giant polytene chromosomes are paired in interphase and often separate from each other during the preparation at the nonhomologous sites. Without induction, no Moe staining can be observed in the corresponding region, but after heat shock, we found an extra Moe-positive band at this chromosomal location though only on one of the homologues (Fig. 7D). This suggests that Moe participates in $h s p$ gene transcription and not the construction of the puff structure. To confirm this finding, we tested whether heat-shock puffs are formed in the moe $e^{\text {NES }}$ mutant. Staining for polymerase II CTD phospho-serine 5 (Pol2-PS5) on heat-shocked polytenic chromosomes prepared from moe $e^{\mathrm{NES}}$ mutant salivary glands showed that puffs can develop after heat shock (Fig. 7E). The results of the chromosome staining experiments together with the mRNA-seq results prove that Moe participates in heat-shock gene transcription.

\section{Discussion}

To attain deeper knowledge about the nuclear functions of the Drosophila ERM protein, Moe, we tagged the moe gene in situ with an NES sequence and analyzed the consequences of the lack of nuclear Moe. The moe $e^{\text {NES }}$ mutants exhibited diverse phenotypes that were of both zygotic and maternal origin. Maternal-effect phenotypes included posterior developmental malformations, dominant sterility, and lethality at embryonic and larval stages. Decreased life span, male genitalia rotation, reduced climbing ability, lethality at embryonic and larval stages, and decreased heat stress tolerance are zygotic phenotypes. Interestingly, decrease in viability at 18 and $25^{\circ} \mathrm{C}$ and in climbing activity was observed in $m o e^{\mathrm{NES}}$ mutant males only. Considering that the moe gene is on the first chromosome, these sex-specific phenotypic differences are 

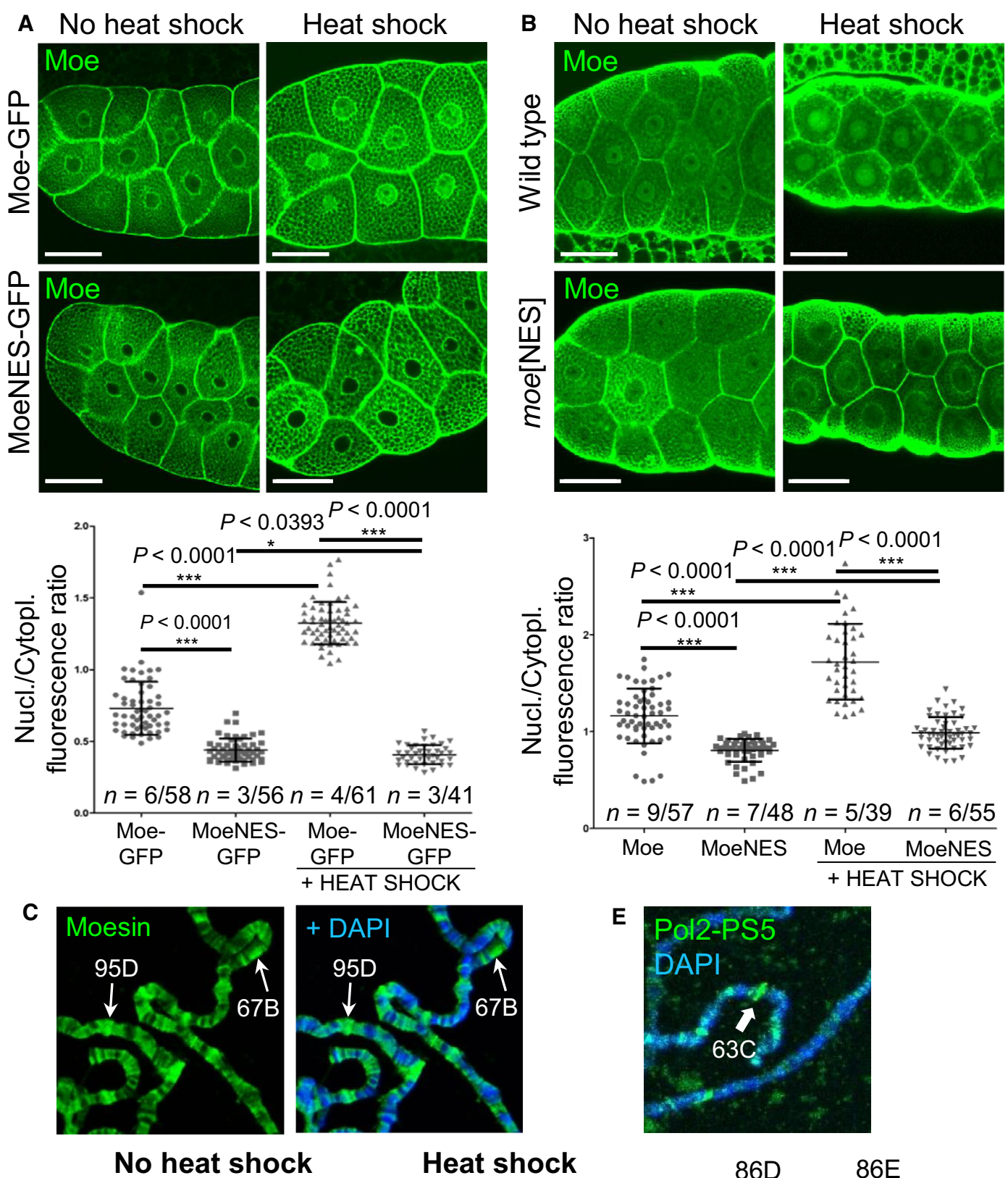

Heat shock

Inactive, heat shock Transcription without inducible transgene at $86 \mathrm{E}$ puff formation at $86 \mathrm{E}$
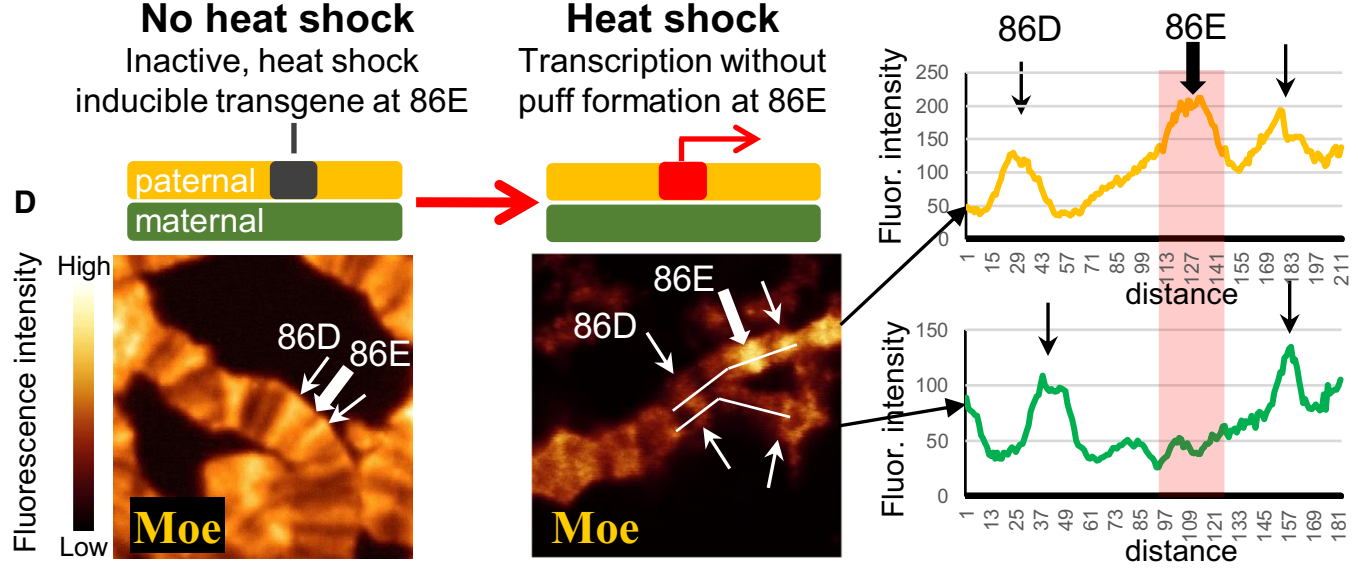
Fig. 7. Moe is not required for the formation of the chromosome heat-shock puff structure. (A-B) The relative level of the MoeNES protein is significantly lower in the nuclei of larval salivary gland cells both at $25^{\circ} \mathrm{C}$ and under heat shock $\left(37^{\circ} \mathrm{C}\right)$ as revealed by immunostaining for transgenically expressed MoeNES-GFP (GFP antibody — green) (A) and endogenous MoeNES (Moe antiserum-green) (B) proteins. Representative images of two independent experiments. $n-$ is the total number of salivary glands and cells examined, respectively. $w^{1118}$ flies were used as WT in (B). Error bars $=\mathrm{SD}, * P<0.05, * * * P<0.0001$ (unpaired $t$-test). Scale bars, $50 \mu \mathrm{m}$. (C) Moe accumulates in the heat-shock puff sites (indicated by white arrows) at cytological locations 67B2: hsp26, 67B3: hsp23, and 95D: hsp68. Representative picture of one of four independent experiments. (D) Heat scale images demonstrating that Moesin localizes to the hsp gene also in the absence of the puff structure. The heat shock-inducible transgene ( $h s>$ FLP) is inserted at cytological position 86E (thick arrow) but only in one of the homologues. Upon heat shock, the transgene becomes transcriptionally active without puff formation and induces the accumulation of Moe at the site (Moe-HA-yellow). Representative images of five independent experiments. (E) Heat-shock puffs are formed in the moe ${ }^{\mathrm{NES}}$ mutant as revealed by polymerase II CTD phospho-serine5 (Pol2-PS5) staining (green). 63C: hsp82. Representative image of one experiment.

most likely the consequence of dosage compensation. The $\mathrm{X}$ chromosome of hemizygous males presumably produces more MoeNES protein than that of the female, which therefore suggests a dominant-negative effect for the protein in the development of these phenotypes.

The quantitation of Moe and P-Moe levels in the $m o e^{\mathrm{NES}}$ mutant ovary revealed that the phosphorylation, cytoplasmic localization, and intracellular distribution of the MoeNES protein are normal. The experiment also showed that the total Moe protein amount is reduced by about $30 \%$ in the mutant. This, in theory, could contribute to the phenotypes observed in the mutant. However, as shown earlier, the hypomorphic moe allele, EP1652, has a reduced Moe protein level [19] but moe[EP1652] homozygous animals are fertile and viable with no apparent visible phenotype, strongly suggesting that the decreased amount of Moe cannot be responsible for the phenotypes The subsequent control experiments demonstrated that the cytoplasmic functions of Moe are unaffected; therefore, the observed phenotypes are due to the perturbation of nuclear Moe function.

The mRNA-seq experiment revealed that the exclusion of Moe from the nucleus causes altered transcription of nearly 690 genes, from which 371 are significantly upregulated and 315 are downregulated in the mutant. Nuclear Moe was shown to participate in mRNA export [16] but it is conceivable that, similarly to the cytoplasm, Moe has multiple functions in the nucleus. In the case of the genes with decreased expression, Moe most likely plays a positive role in their transcription regulation, while in the case of upregulated genes, the protein is involved in their repression. Alternatively, the block of mRNA export, as a consequence of the lack of nuclear Moe, can also increase the activity of the genes affected, as suggested earlier [16], thereby accounting for the elevation of transcript levels.

The transcriptome analysis was performed on the ovary, but it is a reasonable assumption that part of these gene expression changes is not specific to the ovary. Moe is almost ubiquitously expressed in the animal at all developmental stages; therefore, the lack of nuclear Moesin should affect gene expression in other tissues as well. On the other hand, most of the genes with altered expression in the moeNES mutant are active in many other tissues throughout development. In fact, for example, the analysis of the heatshock genes provides supporting evidence for this assumption, because decreased heat stress tolerance was observed in the adults.

The mRNA-seq data showed that in moe $e^{\mathrm{NES}}$ mutant animals, the transcription of three key regulators of development, Notch, dpp, and vasa, is disturbed. Notch is a transmembrane receptor of a highly conserved cell signaling system which plays major role in the regulation of development. Notch mutants in Drosophila have characteristic wing margin 'notches', a phenotype also manifested in moe $e^{\mathrm{NES}}$ mutants (Fig. 3B). Dpp is a secreted morphogen necessary for the correct patterning and development of the early embryo. It has been shown recently that it is also essential for the maintenance of primordial germ cell identity [33]. Vasa is an evolutionarily conserved ATP-dependent RNA helicase that plays central role in germ cell determination and function [34]. Our microscopy experiments demonstrated that despite altered vasa expression in moe $e^{\text {NES }}$ mothers and $100 \%$ sterility in their progenies, the Vasa protein localizes properly at the posterior pole of the developing embryo laid by moe $e^{\mathrm{NES}}$ mothers (Fig. 2D). Interestingly, we also found that the Vasa signal vanishes in the embryonic germ cells, and as a consequence, all germ cells disappear after cellularization in the embryos of moe $e^{\text {NES }}$ mothers (C.B. unpublished result). Future experiments should shed light on the role of Dpp and Vasa in the molecular mechanism responsible for germ cell loss in $m o e^{\mathrm{NES}}$ progenies.

As a consequence of the changes in transcript levels, different molecular mechanisms can be responsible for the development of the observed phenotypes. For instance, the altered activity of the apoptotic 
machinery or sex-specific transcription factors can lead to genitalia rotation in males [35]. The complete loss of pole cells observed in the F1 progeny can be due to uncontrolled transposon activity [36], impaired sumoylation [37], or compromised BMP signaling [38]).

The exploration of the causes behind the various phenotypes was beyond the scope of this study but our mRNA-seq data uncovered that the transcription of a group of heat shock-inducible hsp genes is significantly reduced in the moe $e^{\mathrm{NES}}$ mutants. Drosophila hsp genes encode protein chaperones with versatile functions such as thermotolerance, normal life span, fertility and germline stem cell maintenance, locomotor activity, and developmental timing. Therefore, it is tempting to speculate that the low level of $h s p$ gene transcription alone can contribute to most of the phenotypes observed in the moe NES mutant. Although the lack of nuclear Moe does not fully eliminate $h s p$ gene expression, the combination of reduced expression of multiple $h s p$ genes can account for the severity of the phenotypes observed. Given this possibility, as well as the findings that during heat stress Moe accumulates in the nucleus at the $h s p$ loci and that moe $e^{\mathrm{NES}}$ mutants exhibit reduced thermotolerance, we propose that in the nucleus Moe is required for the normal expression of heat-shock genes. The mechanism through which Moe contributes to $h s p$ transcription is still an open question. No DNA-binding ability of Moe has been described; therefore, the association of Moe with the $h s p$ genes is most likely indirect. An obvious candidate for a binding partner of Moe is nuclear actin, which plays important role in transcription [1,39]. However, the exact activity Moe performs in the nucleus during hsp gene transcription has to be explored in the future. We believe that our results open up a new avenue to understanding the ERM protein family, their exact functions in growth and differentiation, and contribute to a better understanding of the transcription regulation of heat-shock genes.

\section{Materials and methods}

\section{Fly stocks}

Fly strains were maintained, and crosses were carried out on standard cornmeal, yeast, and sucrose Drosophila medium at $25^{\circ} \mathrm{C}$. Stocks number $51324\left(w^{1118}\right.$; PBac $\{y[+\mathrm{mDin}-$ $\mathrm{t} 2]=v a s-C a s 9\} \mathrm{VK} 00027), \quad 42278 \quad\left(w^{1118} ; \quad\right.$ PBac $\{$ Med GFP.FLAG $\}$ VK00037), $12015 \quad\left(w^{67} c^{23} \quad \mathrm{P}\{\mathrm{w}+\mathrm{mC}=\mathrm{lacW}\}\right.$ $\left.m o e^{\mathrm{G} 0415} / \mathrm{FM} 7 \mathrm{c}\right)$ and 279 ( $w^{1118} ; \mathrm{MKRS} ; \mathrm{P}\{\mathrm{hsFLP}\} 86 \mathrm{E} /$ TM6B, Tb) were obtained from the Bloomington Drosophila Stock Center (Indiana University, Bloomington, IN, USA). The $D p(1 ; Y) 619, y[+] B^{S} / w^{l} o c^{9} / C(1) D X, y^{l} f^{l}$
(No. 108358) stock was obtained from Kyoto Stock Center (https://kyotofly.kit.jp; Institute of Technology, Kyoto, Japan). The attached $\mathrm{X}$ and vasa:AID:EGFP lines were provided by Miklós Erdélyi (BRC, Szeged, Hungary), and the oskMS2-MS-GFP and $w, \mathrm{P}\left\{w^{+}\right.$, matTub4:GFP-Staufen $\}$; $\operatorname{stau}^{\mathrm{D} 3} / \mathrm{CyO}$ stocks were kind gifts from Daniel St Johnston (University of Cambridge, UK). The transgenic lines expressing Moe-GFP and Moe-HA have been described previously [16].

To generate flies expressing NES and GFP (MoeNESGFP)-tagged full-length Moe, the coding region was PCR amplified from the cDNA SD10366 (DGC Gold collection, BDGP) using the primer pair Moe_cDNSFw and MoeNES_cDNSRev with gateway recombination sites. The resulting PCR product was sequence-verified and recombined into pDONR221 plasmid, then subcloned into the pPWG gateway vector (Drosophila Gateway Vector Collection) which enables P-element-based random integration into the fly genome. The construct was sequence-verified, and standard Drosophila methods were used to generate transgenic flies. Moe_cDNSFw: 5'-GGG GAC AAG TTT GTA CAA AAA AGC AGG CTT CAC CAT GTC TCC AAA AGC GCT A-3'; MoeNES_cDNSRev (NES-tag underlined): 5'-GGGG AC CAC TTT GTA CAA GAA AGC TGG GTC TGT CTT GTT GAT ATC AAG ACC TGC TAA TTT CAA GGC TAA TTC ATT GCT GGA CAT GTT CTC AAA C-3'.

\section{Drosophila ovary dissection and in vivo microscopy}

Flies were dissected in PBS (137 mm NaCl, $2.7 \mathrm{~mm} \mathrm{KCl}$, $10 \mathrm{~mm} \mathrm{Na} \mathrm{HPO}_{4}, 1.8 \mathrm{mM} \mathrm{KH}_{2} \mathrm{PO}_{4}$ ), and the ovaries were transferred to a microscope slide with a drop of Drosophila Schneider's medium (Lonza, Basel, Switzerland, Schneider's Drosophila Medium, Modified, 04-351Q), then covered with a glass coverslip. The samples were imaged with an Olympus confocal microscope (Olympus Europa SE \& Co. KG, Hamburg, Germany, Olympus Fluoview FV1000 Confocal Microscope, $40 \times$ oil immersion objective, $1.3 \mathrm{NA}$ ).

\section{Moesin antisera}

GST-tagged FERM domain of the Drosophila Moe protein was expressed by the tac promoter-IPTG system using the pGEX-6P-1 vector and the BL21 E. coli strain. The protein was purified on Glutathione Sepharose 4B beads (GE Healthcare, Chicago, IL, USA, 17-0756-01); then, high level of soluble FERM-Moe protein was eluted from the beads by the PreScission protease (GE Healthcare, 27-0843-01) and used to immunize three rabbits, yielding three polyclonal sera ( $\alpha$ FERM-Moe1, $\alpha$ FERM-Moe2, $\alpha$ FERMMoe3). All animal experimentation protocols used in this study were performed in accordance with the European animal experimentation and ethics guidelines and have been 


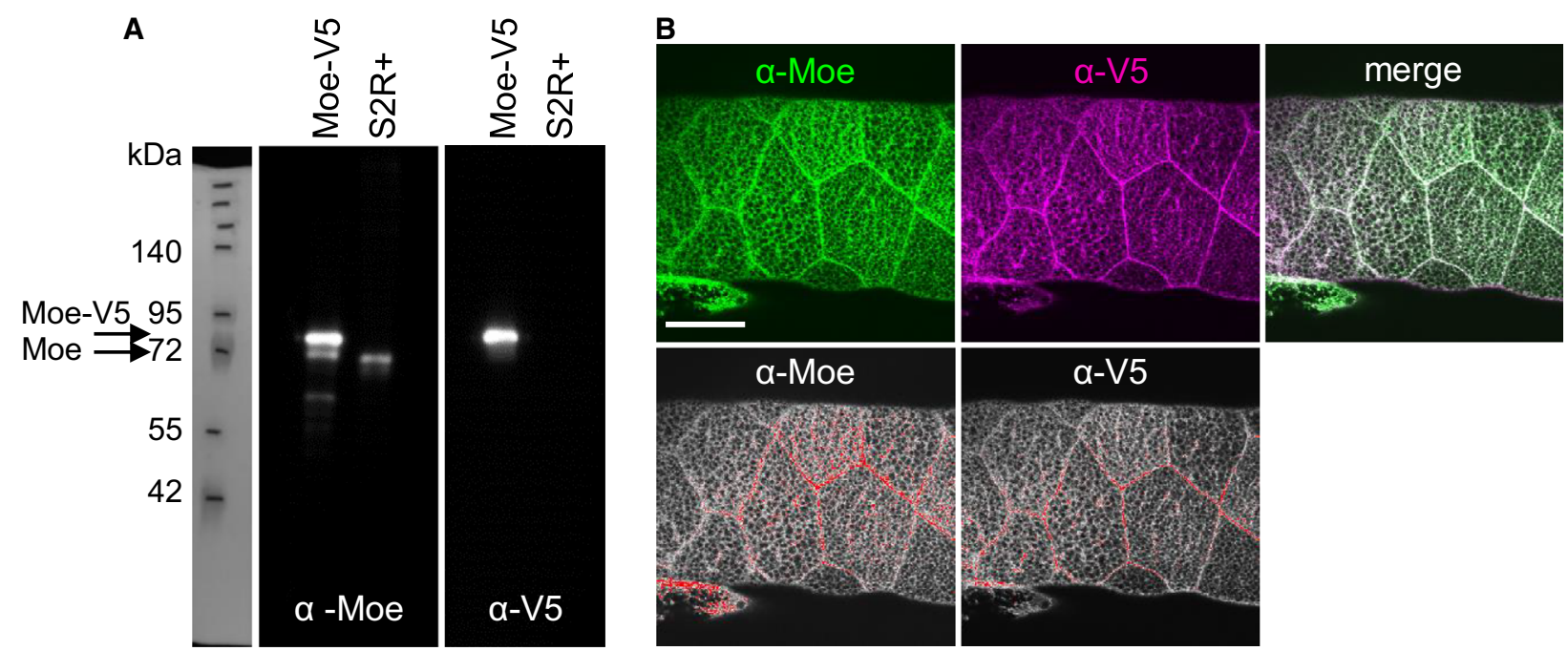

Fig. 8. The Moesin antisera is specifically recognizing the Moe protein. (A) Western blot image showing the endogenous Moe protein and V5-tagged Moe (Moe-V5) expressed from a transgene in Drosophila S2R + cells. The experiment was performed three times; 10 animals were used each genotype. (B) Confocal microscopic image of a salivary gland expressing Moe-V5 and stained for the $V 5$ tag ( $\alpha-V 5)$ and Moesin ( $\alpha$-Moe) to demonstrate the specificity of the anti-Moe antibody in immunostaining. In the images of the bottom row progressively higher fluorescence levels are black, gray, and white, and overexposed pixels are red. Representative pictures of one of two independent experiments. $n \geq 6$ salivary glands in each experiment. Scale bar, $50 \mu \mathrm{m}$.

approved by the local Animal Experimentation and Ethics Committee of the Biological Research Centre, Szeged (MÁB) and by the National Animal Experimentation and Ethics Board (clearance number: XVI./5235/2016). Western blots of the lysate of cultured S2R + Drosophila cells were probed with a serial dilution of all three sera using the ECL system (Millipore, Darmstadt, Germany, Immobilon Western, WBKLS0100). To confirm specificity, V5-tagged, full-length Moe protein was detected with V5 antibody in parallel with the Moe antisera (Fig. 8A). Salivary glands expressing Moe-V5 were stained together with anti-V5 antibody and the Moe antisera to test for specificity in immunostaining (Fig. 8B). Aliquots of the serum number 1 ( $\alpha$ FERM-Moe1) were used in the experiments.

\section{Immunohistochemistry}

After dissections, samples were fixed, permeabilized in PBT $(\mathrm{PBS}+0.1 \%$ Triton $\mathrm{X}-100)$ for $20^{\prime}$, and unspecific reactions were blocked in PBT-N solution (PBT, 1\% BSA, 5\% FCS) for $1 \mathrm{~h}$ at room temperature (RT). Incubation with the primary antibodies was carried out overnight $(\mathrm{O} / \mathrm{N})$ at $4{ }^{\circ} \mathrm{C}$ in PBT-N. After washing (PBS, $2 \times 30 \mathrm{~min}$ at RT), the secondary antibody was applied for $1 \mathrm{~h}$ at RT, and then, the samples were washed, transferred to microscope slides, mounted in Fluoromount-G medium (Thermo Fisher Scientific, Waltham, MA, USA, 00-4958-02), and imaged with an Olympus confocal microscope (Olympus Fluoview FV1000 Confocal Microscope, $63 \times$ oil immersion objective, 1.3 NA).
Salivary gland dissection, heat shock, and immunostaining

L3-stage larvae were heat shocked in a water bath at $37{ }^{\circ} \mathrm{C}$ for $30 \mathrm{~min}$, and then, the salivary glands were dissected in Drosophila Schneider's medium. The samples were fixed in $4 \%$ paraformaldehyde-PBS for $10 \mathrm{~min}$ at RT. After washing and blocking, the immunostaining was carried out with the primary antibodies anti-GFP (1: 500; Thermo Fisher Scientific, A6455), rabbit anti-Moe (1:1000), anti-V5 (1 : 500; Thermo Fisher Scientific R960-25), and anti-phospho-ERM (1 : 200; Cell Signaling Technology, Danvers, MA, USA, Cat. No. 3141) antibodies. After washing in PBS for $30 \mathrm{~min}$, the samples were incubated with Alexa Fluor 488 labeled anti-rabbit antibody (1:600; Thermo Fisher Scientific, A11008). Samples were washed, mounted, and imaged as described above.

\section{Immunostaining of embryos}

Embryos of synchronized females $\left(w^{1118}\right.$ and $\left.m o e^{\mathrm{NES}} / w^{1118}\right)$ were collected $1-2 \mathrm{~h}$ after egg-laying and were dechorionated in $50 \%$ bleach for $2 \mathrm{~min}$. The samples were washed with PBS for $5 \mathrm{~min}$, fixed in formaldehyde-saturated heptane (1: $1-35 \%$ formaldehyde:heptane) solution. The fixative was replaced with PBS-Tween (PBS $+0.1 \%$ Tween20), and the embryos were devitellinized manually with microdissecting needles. After devitellinization, samples were blocked and incubated with the anti-Vasa (1:200; DSHB, University of Iowa, Iowa City, IA, USA, Cat. No. 
760351) primary antibody. After washing, anti-rat Alexa Fluor 647 (1 : 600; Thermo Fisher Scientific, A21247) secondary antibody was used together with DAPI (SigmaAldrich, St. Louis, MO, USA, Cat. No. D9564, final cc. $0.2 \mathrm{~g} \cdot \mathrm{mL}^{-1}$ in PBT-N). The embryos were washed with PBT (PBS, $0.1 \%$ Triton X-100) for $2 \times 30 \mathrm{~min}$ at RT, mounted, and imaged.

\section{Immunostaining of ovaries}

Dissection of females was performed in PBS, and the ovaries were placed into $4 \%$ paraformaldehyde-PBS for $20 \mathrm{~min}$ at RT. The ovaries were then partially dissected to ovarioles in PBS and washed in PBT for $2 \times 30 \mathrm{~min}$ at RT. Ovarioles were blocked and incubated with the anti-phospho-ERM (1 : 200; Cell Signaling Technology, 3141) primary antibody. After washing with PBT for $2 \times 30 \mathrm{~min}$ at RT, the samples were incubated with anti-rabbit Alexa Fluor 488 (1: 600; Thermo Fisher Scientific, A11008) secondary antibody, DAPI and Phalloidin Alexa Fluor 546 (1 : 40; Thermo Fisher Scientific, A22283). Wheat Germ Agglutinin (WGA) Alexa Fluor 647 (Thermo Fisher Scientific, W32466) was used to label the cell membrane. WGA staining was performed in PBS (1: 500) for 45 min before blocking step in PBT-N. The ovarioles were washed, mounted, and imaged as described above.

\section{Polytene chromosome preparation}

Dissected salivary glands were fixed in $45 \%$ acetic acid-PBS for $5 \mathrm{~min}$ at RT then squashed on a Poly-L-Lysine-coated (Sigma-Aldrich P8920) slide under coverslip. The slide was dipped in liquid nitrogen, and the coverslip was immediately removed with a blade. Blocking and immunostaining were carried out in humidity chamber. Primary antibodies were as follows: anti-Moe (1:1000, rabbit polyclonal), anti-Polymerase II CTD (phospho-serine5, 1 : 200; Abcam, Cambridge, UK, Cat. No. ab5131), anti-HA (1 : 100; Sigma-Aldrich GW22511), and DAPI $\left(0.2 \mu \mathrm{g} \cdot \mathrm{mL}^{-1}\right.$; SigmaAldrich). After washing $2 \times$ with $300 \mathrm{~mm} \mathrm{NaCl}$ containing $0.2 \%$ Tween-20 for $10 \mathrm{~min}$ at RT, samples were incubated with the fluorescently labeled secondary antibody (1:600; Invitrogen, Carlsbad, CA, USA) for $2 \mathrm{~h}$ in dark at RT. Squash preparations were mounted in $20 \mu$ Fluoromount$\mathrm{G}$ medium (Southern Biotech, Birmingham, AL, USA) and imaged with a Leica TCS SP5 Confocal Microscope using a $63.0 \times 1.40$ oil objective.

\section{Western blot}

Cell lysates of dissected ovaries or cultured Drosophila $\mathrm{S} 2 \mathrm{R}+$ cells were used. Before loading the samples on the gel, $1 \mu \mathrm{L}$ beta-mercaptoethanol was added and the samples were boiled for $5 \mathrm{~min}$ and spun for $5 \mathrm{~min}$ with $18000 \mathrm{~g}$ at
RT. $10 \%$ acrylamide gel was used; the proteins were transferred for $1 \mathrm{~h}$ to Polyvinylidene fluoride (PVDF) membrane (Millipore Transfer Membranes Immobilon-P, Cat.no.: IPVH00010 PVDF $0.45 \mu \mathrm{m}$ ) using $300 \mathrm{~mA}$ at $4{ }^{\circ} \mathrm{C}$. Blocking was performed in 5\% milk powder-TBST (25 mM Tris, $150 \mathrm{~mm} \mathrm{NaCl}, \mathrm{pH} 7.5+0.1 \%$ Tween-20) for $1.5 \mathrm{~h}$, and then, the membrane was incubated with the primary antibodies anti-Moe (rabbit polyclonal, $1: 100$ 000), anti-V5 (1 : 5000; Thermo Fisher Scientific R960-25), and antiActin (mouse monoclonal; Sigma-Aldrich, MAB1501, 1 : 5000) $\mathrm{O} / \mathrm{N}$ at $4{ }^{\circ} \mathrm{C}$. The membrane was washed $1 \times$ in TBST for $30 \mathrm{~min}$ and incubated with the HRP-conjugated secondary antibody for $60 \mathrm{~min}$. After washing three times for $30 \mathrm{~min}$ with TBST at RT, the signal was visualized using HRP Substrate solution (Millipore Immobilon Western Chemiluminescent HRP Substrate, Cat. No.: WBKLS01000) and the films were developed.

\section{Physiological tests}

In all of these experiments, flies were reared on standard Drosophila medium at constant temperature $\left(25^{\circ} \mathrm{C}\right)$. In the hatching rate study, the number of hatched embryos, eclosed larvae and unhatched pharate adults was determined in a 2-week-old culture. For the statistical analysis of the data, we calculated mean values, standard deviations (SD), and performed unpaired $t$-tests to determine the significance of difference.

\section{Embryonic lethality}

Groups of 30 females of $m o e^{\mathrm{G} 0415} / w^{1118}, m o e^{\mathrm{NES}} / w^{1118}$, and $m o e^{\mathrm{NES}} /$ moe $^{\mathrm{G} 0415}$ genotypes were collected on the same morning and crossed to $w^{1118} / \mathrm{Y}$ males. Embryos were lined up every day in groups of 100 on black agar plate, and the hatched larvae, and unhatched eggs were counted.

\section{Larval lethality}

$m o e^{\mathrm{G} 0415} / w^{1118}, \quad m o e^{\mathrm{NES}} / w^{1118}$, and $m o e^{\mathrm{NES}} / m o e^{\mathrm{G} 0415}$ females of the same age were crossed to $w^{1118} / \mathrm{Y}$ males. After the synchronization of egg-laying, hatched larvae in groups of 20 were placed in separate vials containing fresh fly food. The number of 3rd-instar larvae, pupae, and hatched flies was recorded in the following 2 weeks.

\section{Lifespan analysis}

When testing the viability of adult animals, virgin males $\left(w^{1118} / \mathrm{Y}\right.$ and $\left.m o e^{\mathrm{NES}} / \mathrm{Y}\right)$ and virgin females $\left(m o e^{\mathrm{G} 0415} /\right.$ $w^{1118}, m o e^{\mathrm{NES}} / w^{1118}$, and $\left.m o e^{\mathrm{NES}} / m^{\mathrm{G} 0415}\right)$ of the same age were tested separately in groups of five. In each experiment, 30 males and 30 females were analyzed by this way. The flies were transferred to fresh medium every day, and the 
number of living flies was recorded. This experiment was performed at $18{ }^{\circ} \mathrm{C}$ and at $29^{\circ} \mathrm{C}$ as well. During the experiment designed to rescue the decreased viability of $m o e^{\mathrm{NES}}$ mutants, 30 virgin females were crossed individually to five $w^{1118}$ males, transferred to fresh medium every day, and the number of living flies was recorded.

\section{Starvation test}

During the starvation test, $30 \mathrm{moe}^{\mathrm{NES}}$ mutant and 30 control females were reared individually together with 3 male siblings. Flies were transferred to fresh medium every day, and the number of eggs deposited was counted. On the third and fourth days, sucrose starvation medium $(1 \%$ agar, $0.5 \%$ propionic acid - phosphoric acid (9:1), 5\% sucrose) was used [40].

\section{Fertility test}

To determine the egg yield of $m o e^{\mathrm{NES}}$ mutant flies, $m o e^{\mathrm{G} 0415} / w^{1118}, m^{\mathrm{NES}} / w^{1118}$, and $m o e^{\mathrm{NES}} / m_{0} e^{\mathrm{G} 0415}$ virgin females were collected and crossed individually to $w^{1118} / \mathrm{Y}$. The flies were transferred to fresh medium every day, and the eggs were counted for 12 days.

\section{Climbing assay}

To test the climbing capability, $w^{1118} / \mathrm{Y}$ and $m o e^{\mathrm{NES}} / \mathrm{Y}$ virgin males were collected and kept at normal medium for 1 day or in other experiments for a week. Males were transferred to a transparent plastic vial with a centimeter scale, and the number of flies that were able to cover the 15-cm distance in $8 \mathrm{~s}$ was determined [41].

\section{In situ gene tagging with CRISPR-Cas9}

\section{Generation and testing of gRNAs}

To search for gRNA target sites in the moe gene, we used the flyCRISPR Optimal Target Finder online software (http://targetfinder.flycrispr.neuro.brown.edu/) and selected four target sites: Cas9_1: 5'-CCAATGAATATAGTCTTGATACC-3'; Cas9_2: 5'-CCCTTATGCTCCACTCAACTAAC-3'; Cas9_3: 5'-CCTATGTAATATACTGGAAA CTT-3'; Cas9_4: 5'-CCAACCGCGCAACTTAAGGTA GC- $3^{\prime}$. The sense and antisense sequences of the target sites were synthesized with overhangs (Integrated DNA Technologies) and cloned into the pU6-BbsI-chiRNA plasmid following the protocol described on the https://flycrispr. org/protocols/grna/ website. The gRNA constructs were injected in pairs into Drosophila embryos expressing Cas9 to test for cleavage efficiency. The injected gRNA pairs were Cas9_1 + Cas9_3, Cas9_1 + Cas9_4, Cas9_2 + Cas9_3, and Cas9_2 + Cas9_4. The resulting deletions were detected by PCR and gel electrophoresis according to the protocol at https://flycrispr.org/protocols/embryonic-pcr/. The $5^{\prime}$-GCATTCAGGACGAGGTCAACG-3' forward and 5'-GCCTCTCAATCGAGATATACAACT-3' reverse primers were used in the PCR reaction. The most efficient gRNA pair was Cas9_2 + Cas9_3, as demonstrated by the expected 1132 bp deletion.

\section{Construction of repair template}

To generate the pHD-DsRed-MoeNES repair template, the genomic region (X:8871181-8875777) containing the entire moe gene was PCR amplified from $w^{1118}$ flies with the $5^{\prime}$ GCTGATCCAGGACATTACACTG- $3^{\prime}$ forward and $5^{\prime}$ GATCACGCTGTGAACTCTGC- $3^{\prime}$ reverse primers. The $\sim 5 \mathrm{kbp}$ PCR product was cloned into pJET vector (CloneJET PCR Cloning Kit; Thermo Fisher Scientific, K1231) according to the manufacturer's instructions. To protect the repair template from unintended Cas9 cleavage, the Cas9_3 gRNA target site has been modified in the PCR product with site-directed mutagenesis (Quickchange II Site-Directed Mutagenesis KIT; Agilent, Santa Clara, CA, USA, Cat No. 200523). The mutagenic primers were 5'TAAAACAACAAAATGCCACGTTTTTATTTTATCTATATAATATACTGGAAACTTTCGACATGT-3' forward and 5'-ACATGTCGAAAGTTTCCAGTATATTATATAGATAAAATAAAAACGTGGCATTTTGTTGTTTTA-3'

reverse primers (mutated nucleotides are marked in red). Next, the repair template construct has been assembled from three PCR products amplified with the following primer pairs (restriction sites are underlined): 5'-CGTAGAATTCATAGCTGATCCAGGACATTACACTG-3' forward (Fw_Moe_EcoRI) and 5'-GGAGGCGGCCGC AGACATAAGGGACTCGATTTCGAAC-3' reverse (Rev_Moe_NotI) primers for PCR1, 5'-GCATGCTCTTC GTATCTCCACTCAACTAACAATATCA-3' forward (Fw_Moe_SapI) and 5'-ATAAAGCTTTTATGTCTTGTTGATATCAAGACCTGCTAATTTCAAGGCTAATTCAT TCATGTTCTCAAACTGATCGACG-3' reverse (Rev_ HindIII) primers (NES is highlighted in red) for PCR2, and 5'-GGCGAAAGCTTAAGCTATCAAAGATCAGAGATC-3' forward (Fw_Moe_HindIII) and 5'-GAAT GCTCTTCAGACCAATCTGTTGATGTAGGC-3' reverse (Rev_Moe_SapI) primers for PCR3. After digestion with EcoRI and NotI, the PCR1 product was cloned into the pHD-DsRed vector, and then, the PCR2 and PCR3 products were subsequently cloned into the pHD-DsRedPCR1 construct with HindIII and SapI digestions.

\section{The generation of moe $e^{\mathrm{NES}}$ mutant fly lines}

The pU6-BbsI-chiRNA plasmids $\left(150-150 \mathrm{ng} \cdot \mu \mathrm{L}^{-1}\right)$ and the repair template plasmid $\left(550 \mathrm{ng} \cdot \mu \mathrm{L}^{-1}\right)$ were mixed and injected into $w^{1118} ; \operatorname{PBac}\{\mathrm{y}[+\mathrm{mDint} 2]=$ vas-Cas 9$\} \mathrm{VK} 00027$ embryos. The progenies of the injected flies were screened 
for eye-specific expression of the DsRed marker gene. Successful in vivo tagging was confirmed by sequencing the corresponding region in the moe gene and the moe $e^{\mathrm{NES}}$ mRNA.

\section{Scanning electron microscopy}

Adult flies were fixed with $2.5 \%(\mathrm{v} / \mathrm{v})$ glutaraldehyde and 0.05 M cacodylate buffer $\mathrm{pH} 7.2$ in PBS (pH 7.4) overnight. Samples were then washed twice with PBS and dehydrated with a graded ethanol series $(30 \%, 50 \%, 70 \%, 80 \%$, and $100 \%$ ethanol, each for overnight). The samples were dried with a critical point dryer, followed by $12-\mathrm{nm}$ gold coating (Quorum Technologies, Laughton, East Sussex, UK) and observed under a field emission JEOL JSM-7100F/LV scanning electron microscope (JEOL Ltd., Tokyo, Japan).

\section{Imaging of adult body parts}

Adult flies were anesthetized with diethyl ether and arranged under the microscope (Lecia MZFL III). Images with different focal planes were focus stacked using the CombineZP freeware.

\section{mRNA-Seq library preparation, sequencing, and analysis}

Total RNA preparation was performed from three replicates of 3-day-old fly ovaries of 10 females/genotype $\left(m o e^{\mathrm{G} 0415} /\right.$ $w^{1118}, m o e^{\mathrm{NES}} / w^{1118}$, and $m o e^{\mathrm{NES}} / m o e^{\mathrm{G} 0415}$ ) by using the TRIzol method as described in Ref. [16]. The mRNA-seq library was prepared using the TruSeq Stranded mRNA Library Prep Kit (Illumina, San Diego, CA, USA) and sequenced with the HiSEq 2500 sequencing platform (performed at the NYUAD Sequencing Center). All of the subsequent analyses, including quality trimming, were executed using the BioSAIL workflow execution system. The raw reads were quality trimmed using Trimmomatic (version 0.36 ) to trim low-quality bases, systematic base-calling errors, as well sequencing adapter contamination. FastQC was used to assess the quality of the sequenced reads pre/postquality trimming. Only the reads that passed quality trimming in pairs were retained for downstream analysis. The quality trimmed mRNA-seq reads were aligned to the D. melanogaster genome using HISAT2 (version 2.0.4) (http:// daehwankimlab.github.io/hisat2/). The resulting SAM alignment files for each sequenced sample were then converted to BAM format and sorted by coordinate using SAMTOOLs (version 0.1.19) (http://www.htslib.org/). The BAM alignment files were processed using HTseq-count using the reference annotation file to produce raw counts for each sample. The raw counts were then analyzed using the online analysis portal NASQAR (http://nasqar.abudhabi.nyu.edu/) in order to merge, normalize, and identify differentially expressed genes. Differentially expressed genes by at least twofold $\left(\log _{2}\right.$ (FC) $\geq 1$ and adjusted $P$-value of $<0.05$ for upregulated genes, and $\log _{2}(\mathrm{FC}) \leq-1$ and adjusted $P$-value of $<0.05$ for downregulated genes between the genotypes $m o e^{\mathrm{G} 0415} / w^{1118}$, $m o e^{\mathrm{NES}} / w^{1118}$, and $m o e^{\mathrm{NES}} / m^{\mathrm{G} 0415}$ were subjected to $\mathrm{GO}$ database of Flybase (https://flybase.org/). Venn diagram was produced by using the 'Venn Diagrams' freeware developed by the Van de Peer Lab at the University of Gent, Belgium (http://bioinformatics.psb.ugent.be/webtools/Venn/). The RNA-seq data set has been deposited in the public repository GEO and can be retrieved using accession number GSE155778.

\section{RT-qPCR experiments}

In the RT-qPCR experiments, five pairs of ovaries from females of $m o e^{\mathrm{G} 0415} / w^{1118}, m o e^{\mathrm{NES}} / w^{1118}$, and $m o e^{\mathrm{NES}} /$ $m o e^{\mathrm{G} 0415}$ genotypes were dissected in ice-cold PBS. The ovaries were transferred to Drosophila Schneider's medium (Lonza, Schneider's Drosophila Medium, Modified, 04351Q), incubated at RT or heat shocked at $37^{\circ} \mathrm{C}$ for $30 \mathrm{~min}$, and dipped into liquid nitrogen. Zymo ResearchQuick-RNA Miniprep Kit (Thermo Fisher Scientific) was used to purify total RNA. For the first-strand cDNA synthesis, RevertAid First-strand cDNA synthesis Kit (Thermo Fisher Scientific) was used according to manufacturer's instruction, using $2 \mu \mathrm{g}$ of total RNA and random hexamer primers. Maxima SYBR Green/ROX qPCR Master Mix $(2 \times)$ (Thermo Fisher Scientific) was used for the real-time quantitative PCR, following the manufacturer's instructions. Reactions were run in triplicates in Rotor-Gene Q (Qiagen, Hilden, Germany) qPCR platform with the following reaction conditions: $95^{\circ} \mathrm{C} 10 \mathrm{~min}, 45$ cycles of $95{ }^{\circ} \mathrm{C} 10 \mathrm{~s}, 54{ }^{\circ} \mathrm{C} 15 \mathrm{~s}$, and $72{ }^{\circ} \mathrm{C} 20 \mathrm{~s}$. rp 49 -specific primers were used as internal control in the PCR. RT-qPCR data analysis was performed with Rotor-Gene Q Series Software and Q-Rex 1.0. List of primers used in quantitative RT-PCR: rp49-Fw: 5'-TCGTGAAGAAGCGCACCAAG-3'; rp49-Rev: 5'-CTTGAAGCGGCGACGCAC-3'; qHsp70Aa-Fw: GGATTTGGAGGCTACTCTGGA; qHsp 70Aa-Rev: GATCGAAACATTCTTATCAGTCTC; qHsp 70Ba-Fw: AGCCAACTGTGGCCAACAG; qHsp70Ba-Rev: CTTTTAAAAACTTAAGCCAGGAACTG; qHsp23-Fw: GAGCAGGACAATGGCAACGA; qHsp23-Rev: AGTG TCCTTGGATAACTACAGCT; qHsp26-Fw: TGTGGG CAAGGATGGATTCCA; qHsp26:-Rev: TGGCGCATGA TGTGACCATG; qHsp68-Fw: AACTTTGGACAGCAA GCTGG; qHsp68-Rev: AATATAGGGGGACTTCG TTTGA.

\section{Quantification of pixel intensities and statistical analyses}

Cytoplasmic-nuclear fluorescence intensity measurements were made as described in Ref [42]. In short: Cells were measured once using IMAGEJ (https://imagej/nih.gov/ij/). 
ROIs were drawn by hand in the entire nucleus and cytoplasm. Background fluorescence intensity was subtracted from the data prior to calculating the $\mathrm{N} / \mathrm{C}$ ratio. In every measurement, 8-10 cells per salivary gland and 4-6 cells per egg chamber were examined. The GRAPHPAD PRISM 5 software (GraphPad Software Inc., San Diego, CA, USA) and unpaired $t$-test were used for statistical analysis. Error bar in bar charts indicates SD while in the boxplots represents minimum and maximum values. Statistical significance is marked with $* P<0.05, \quad * * P<0.01$, $* * * P<0.0001$, and n.s. (not significant).

For embryo size measurement, the length and width of 100 embryos of each genotype were measured with the ImageJ freeware. The length-width ratio was calculated for individual embryos, and the statistical analysis was performed with GRAPHPAD PRISM 5. For the quantitation of the protein levels, the signal intensities of individual bands on the western blot image were measured with the UVITEC Alliance software (Cambridge, UK). Data were normalized with the help of the actin loading control, and the ratio between Moe and P-Moe was calculated.

\section{Acknowledgements}

We thank Henrik Gyurkovics (BRC, Szeged) for helpful discussions, Ildikó Velkeyné Krausz and Anikó Berente for technical assistance. Stocks obtained from the Bloomington Drosophila Stock Center (NIH P40OD018537) were used in this study. This work was supported by the Ministry for National Economy of Hungary grants GINOP-2.3.2-15-2016-00001 and GINOP-2.3.2-15-2016-00032 (to PV and ZL), the Hungarian National Research, Development and Innovation Office-NKFIH grants PD127968 (to IK), NKFIH-871-3/2020 (to PV), and K132155 (to RS and $\mathrm{VV}$ ), the Hungarian Academy of Sciences grant LP2017-7/2017 (to ZL), and partly supported by an NYU Abu Dhabi Research Enhancement Fund and a grant from the Swedish Cancer Society (Cancerfonden) (to PP).

\section{Conflict of interest}

The authors declare no conflict of interest.

\section{Author contributions}

PV conceptualized the study; PV and PP contributed to methodology; CB, IK, CA, TV, ZL, TL, PB, ZK, IB, AM, CV, VV, RS, TV, and AF investigated the study; PV wrote-original draft; $\mathrm{CB}$ and PV wrotereview and editing; $\mathrm{PP}$ and $\mathrm{PV}$ involved in project administration; RS, ZL, PP, and PV involved in funding acquisition.

\section{Peer Review}

The peer review history for this article is available at https://publons.com/publon/10.1111/febs.15779.

\section{References}

1 Kristó I, Bajusz I, Bajusz C, Borkúti P \& Vilmos P (2016) Actin, actin-binding proteins, and actin-related proteins in the nucleus. Histochem Cell Biol 145, 373388.

2 Misu S, Takebayashi M \& Miyamoto K (2017) Nuclear actin in development and transcriptional reprogramming. Front Genet $\mathbf{8}, 27$.

3 Kelpsch DJ \& Tootle TL (2018) Nuclear actin: from discovery to function. Anat Rec 301, 1999-2013.

4 Percipalle P \& Vartiainen M (2019) Cytoskeletal proteins in the cell nucleus: a special nuclear actin perspective. Mol Biol Cell 30, 1781-1785.

5 Clucas J \& Valderrama F (2014) ERM proteins in cancer progression. J Cell Sci 127, 267-275.

6 Michie KA, Bermeister A, Robertson NO, Goodchild SC \& Curmi PMG (2019) Two sides of the coin: Ezrin/ Radixin/Moesin and merlin control membrane structure and contact inhibition. Int J Mol Sci 20, 1996.

7 Shabardina V, Kashima Y, Suzuki Y \& Makalowski W (2019) Emergence and evolution of ERM proteins and merlin in metazoans. Genome Biol Evol 12, 37103724.

8 Fehon RG, McClatchey AI \& Bretscher A (2010) Organizing the cell cortex: the role of ERM proteins. Nat Rev Mol Cell Biol 11, 276-287.

9 Kawaguchi K, Yoshida S, Hatano R \& Asano S (2017) Pathophysiological roles of ezrin/radixin/moesin proteins. Biol Pharm Bull 40, 381-390.

10 Peter A (2002) Mapping and identification of essential gene functions on the $\mathrm{X}$ chromosome of Drosophila. EMBO Rep 3, 34-38.

11 van Fürden D, Johnson K, Segbert C \& Bossinger O (2004) The $C$. elegans ezrin-radixin-moesin protein ERM-1 is necessary for apical junction remodelling and tubulogenesis in the intestine. Dev Biol 272, 262276.

12 Bergquist J, Gobom J, Blomberg A, Roepstorff P \& Ekman R (2001) Identification of nuclei associated proteins by $2 \mathrm{D}$-gel electrophoresis and mass spectrometry. J Neurosci Methods 109, 3-11.

13 Batchelor CL, Woodward AM \& Crouch DH (2004) Nuclear ERM (ezrin, radixin, moesin) proteins: regulation by cell density and nuclear import. Exp Cell Res 296, 208-222.

14 Krawetz R \& Kelly GM (2008) Moesin signalling induces F9 teratocarcinoma cells to differentiate into primitive extraembryonic endoderm. Cell Signal 20, 163-175. 
15 Vilmos P, Jankovics F, Szathmári M, Lukácsovich T, Henn L \& Erdélyi M (2009) Live imaging reveals that the Drosophila actin-binding ERM protein, moesin, colocalizes with the mitotic spindle. Eur J Cell Biol 88, 609-619.

16 Kristó I, Bajusz C, Borsos BN, Pankotai T, Dopie J, Jankovics F, Vartiainen MK, Erdélyi M \& Vilmos P (2017) The actin binding cytoskeletal protein Moesin is involved in nuclear mRNA export. Biochim Biophys Acta Mol Cell Res 1864, 1589-1604.

17 Kazgan N, Williams T, Forsberg LJ \& Brenman JE (2010) Identification of a nuclear export signal in the catalytic subunit of AMP-activated protein kinase. $\mathrm{Mol}$ Biol Cell 21, 3433-3442.

18 Gratz SJ, Rubinstein CD, Harrison MM, Wildonger J \& O'Connor-Giles KM (2015) CRISPR-Cas9 genome editing in Drosophila. Curr Protoc Mol Biol 111, 31.2.131.2.20.

19 Jankovics F, Sinka R, Lukácsovich T \& Erdélyi M (2002) MOESIN crosslinks actin and cell membrane in Drosophila oocytes and is required for OSKAR anchoring. Cur Biol 12, 2060-2065.

20 Speck O, Hughes SC, Noren NK, Kulikauskas RM \& Fehon RG (2003) Moesin functions antagonistically to the Rho pathway to maintain epithelial integrity. Nature 421, 83-87.

21 Polosello C, Delon I, Valenti P, Ferrer P \& Payre F (2002) Dmoesin controls actin-based cell shape and polarity during Drosophila melanogaster oogenesis. Nat Cell Biol 4, 782-789.

22 Huelsmann S, Ylänne J \& Brown NH (2013) Filopodialike actin cables position nuclei in association with perinuclear actin in Drosophila nurse cells. Dev Cell 26, 604-615.

23 Gates J, Nowotarski SH, Yin H, Mahaffey JP, Bridges T, Herrera C, Homem CC, Janody F, Montell DJ \& Pfeifer M (2009) Enabled and Capping protein play important roles in shaping cell behavior during Drosophila oogenesis. Dev Biol 333, 90-107.

24 Pierreux CE, Nicolás FJ \& Hill CS (2000) Transforming growth factor $\beta$-independent shuttling of smad4 between the cytoplasm and nucleus. Mol Cell Biol 20, 9041-9054.

25 Miles WO, Jaffray E, Campbell SG, Takeda S, Bayston LJ, Basu SP, Li M, Raftery LA, Ashe MP, Hay RT \& et al. (2008) Medea SUMOylation restricts the signaling range of the Dpp morphogen in the Drosophila embryo. Genes Dev 22, 2578-2590.

26 Solomon JM, Rossi JM, Golic K, McGarry T \& Lindquist S (1991) Changes in $h s p 70$ alter thermotolerance and heat-shock regulation in Drosophila. New Biol 3, 1106-1120.

27 Gong WJ \& Golic KG (2006) Loss of Hsp70 in Drosophila is pleiotropic, with effects on thermotolerance, recovery from heat shock and neurodegeneration. Genetics 172, 275-286.

28 Vos MJ, Carra S, Kanon B, Bosveld F, Klauke K, Sibon OCM \& Kampinga HH (2016) Specific protein homeostatic functions of small heatshock proteins increase lifespan. Aging Cell 15, 217 226.

29 Lynn Zimmerman J, Petri W \& Meselson M (1983) Accumulation of a specific subset of D. melanogaster heat shock mRNAs in normal development without heat shock. Cell 32, 1161-1170.

30 Cobreros L, Fernández-Miñán A, Luque CM, González-Reyes A \& Martín-Bermudo MD (2008) A role for the chaperone Hsp70 in the regulation of border cell migration in the Drosophila ovary. Mech Dev 125, 1048-1058.

31 Xiao C, Mileva-Seitz V, Seroude L \& Robertson RM (2007) Targeting HSP70 to motoneurons protects locomotor activity from hyperthermia in Drosophila. Dev Neurobiol 67, 438-455.

32 Kjærsgaard A, Demontis D, Kristensen TN, Le N, Faurby S, Pertoldi C, Sørensen JG \& Loeschcke V (2010) Locomotor activity of Drosophila melanogaster in high temperature environments: plastic and evolutionary responses. Clim Res 43, 127-134.

33 Deshpande G, Willis E, Chatterjee S, Fernandez R, Dias K \& Schedl P (2014) BMP signaling and the maintenance of primordial germ cell identity in Drosophila embryos. PLoS One 9, e88847.

34 Gustafson EA \& Wessel GM (2010) Vasa genes: emerging roles in the germ line and in multipotent cells. BioEssays 32, 626-637.

35 Coutelis JB, Petzoldt AG, Spéder P, Suzanne M \& Noselli S (2008) Left-right asymmetry in Drosophila. Semin Cell Dev Biol 19, 252-262.

36 Jankovics F, Bence M, Sinka R, Faragó A, Bodai L, Pettkó-Szandtner A, Ibrahim K, Takács Z, SzarkaKovács AB \& Erdélyi M (2018) Drosophila small ovary gene is required for transposon silencing and heterochromatin organization, and ensures germline stem cell maintenance and differentiation. Development 145, dev170639.

37 Talamillo A, Barroso-Gomila O, Giordano I, Ajuria L, Grillo M, Mayor U \& Barrio R (2020) The role of SUMOylation during development. Biochem Soc Trans 48, 463-478.

38 Dansereau DA \& Lasko P (2008) The development of germline stem cells in Drosophila. Germline Stem Cells Methods Mol Biol 450, 3-26.

39 Sokolova M, Moore HM, Prajapati B, Dopie J, Meriläinen L, Honkanen M, Matos RC, Poukkula M, Hietakangas V \& Vartiainen MK (2018) Nuclear actin is required for transcription during Drosophila oogenesis. iScience 9, 63-70. 
40 Terashima J \& Bownes M (2004) Translating available food into the number of eggs laid by Drosophila melanogaster. Genetics 167, 1711-1719.

41 Manjila S \& Hasan G (2018) Flight and climbing assay for assessing motor functions in Drosophila. BioProtocol 8, e2742.

42 Kelley JB \& Paschal BM (2019) Fluorescence-based quantification of Nucleocytoplasmic transport. Methods 157, 106-114.

\section{Supporting information}

Additional supporting information may be found online in the Supporting Information section at the end of the article.

Table S1. mRNA-seq data and $P$-values of down- and upregulated genes. 